\title{
TOPOLOGICAL PROPERTIES OF SEMIGROUP PRIMES OF A COMMUTATIVE RING
}

\author{
CARMELO A. FINOCCHIARO, MARCO FONTANA, AND DARIO SPIRITO
}

\begin{abstract}
A semigroup prime of a commutative ring $R$ is a prime ideal of the semigroup $(R, \cdot)$. One of the purposes of this paper is to study, from a topological point of view, the space $\mathcal{S}(R)$ of prime semigroups of $R$. We show that, under a natural topology introduced by B. Olberding in 2010, $\mathcal{S}(R)$ is a spectral space (after Hochster), spectral extension of $\operatorname{Spec}(R)$, and that the assignment $R \mapsto \mathcal{S}(R)$ induces a contravariant functor. We then relate - in the case $R$ is an integral domain - the topology on $\mathcal{S}(R)$ with the Zariski topology on the set of overrings of $R$. Furthermore, we investigate the relationship between $\mathcal{S}(R)$ and the space $\mathcal{X}(R)$ consisting of all nonempty inverse-closed subspaces of $\operatorname{Spec}(R)$, which has been introduced and studied in 20]. In this context, we show that $\mathcal{S}(R)$ is a spectral retract of $\mathcal{X}(R)$ and we characterize when $\mathcal{S}(R)$ is canonically homeomorphic to $\mathcal{X}(R)$, both in general and when $\operatorname{Spec}(R)$ is a Noetherian space. In particular, we obtain that, when $R$ is a Bézout domain, $\mathcal{S}(R)$ is canonically homeomorphic both to $\mathcal{X}(R)$ and to the space Overr $(R)$ of the overrings of $R$ (endowed with the Zariski topology). Finally, we compare the space $\mathcal{X}(R)$ with the space $\mathcal{S}(R(T))$ of semigroup primes of the Nagata ring $R(T)$, providing a canonical spectral embedding $\mathcal{X}(R) \hookrightarrow \mathcal{S}(R(T))$ which makes $\mathcal{X}(R)$ a spectral retract of $\mathcal{S}(R(T))$.
\end{abstract}

\section{INTRODUCTION AND PRELIMINARIES}

The concept of prime ideal, and the closely related concept of localization, play a fundamental role in commutative ring theory. In the forties of the last century, the concept of prime ideal was introduced in the setting of semigroups, and some analogies and differences between the ring and semigroup theories were pointed out (cf., for instance, [51, [30, and [40). Since a ring $R$ can be also regarded as a semigroup (by considering only the multiplicative structure), it is reasonable to bring back the concept of semigroup prime from semigroups to rings: hence, we define a semigroup prime of a ring $R$ to be a prime ideal of the $\operatorname{semigroup}(R, \cdot)$.

Clearly, every prime ideal of $R$ is also a semigroup prime, but not conversely: the set $\mathcal{S}(R)$ of all semigroup primes of $R$ is in general much larger than the prime spectrum $\operatorname{Spec}(R)$ of $R$. An additional link ties the two concepts: semigroup primes of $R$ turn out to be the complement of saturated multiplicatively closed subsets of $R$ and so they give rise to general ring of fractions, while prime ideals give rise to localizations.

Date: September 13, 2018.

2010 Mathematics Subject Classification. 13A15, 13G05, 13B10, 13C11, 13F05, 14A05, 54A10.

Key words and phrases. Spectral space, spectral map, Zariski topology, constructible topology, inverse topology, semistar operation, semigroup prime, Nagata ring.

This work was partially supported by GNSAGA of Istituto Nazionale di Alta Matematica. The first named author was also supported by a Post Doc Grant from the University of Technology of Graz (Austrian Science Fund (FWF): P 27816). 
Nevertheless, for a long time, semigroup primes of a commutative ring were left out from the mainstream of investigation, even in the natural context of multiplicative ideal theory of rings and integral domains.

Recently, B. Olberding 47 has considered the space $\mathcal{S}(R)$, equipped with a Zariski-like topology, for obtaining new important properties of the spaces of overrings and valuation overrings of an integral domain $R$.

In this paper, we pursue the study of $\mathcal{S}(R)$, mainly from a topological point of view, considering the general case of a commutative ring $R$ with applications to the special case of when $R$ is an integral domain. The relevant topologies that turn out to be useful in our investigation are the hull-kernel topology (classically introduced by Stone [57]) or Zariski topology, the constructible or patch topology (cf. 31, and [36]), with an underlying ultrafilter theoretic approach (cf. [26], 16] and [41) and the inverse topology introduced by Hochster on arbitrary spectral spaces [36] (definitions and properties used in the present paper will be recalled later in this section).

As a starting point, we prove that $\mathcal{S}(R)$, when endowed with the hull-kernel topology, is a new unconventional example of spectral space (after Hochster), that the inclusion map $\operatorname{Spec}(R) \hookrightarrow \mathcal{S}(R)$ is a spectral map, and that the assignment $R \mapsto \mathcal{S}(R)$ induces a contravariant functor. Next, we compare the spectral space $\mathcal{S}(R)$ with the space $\mathcal{X}(R)$ consisting of all nonempty inverse-closed subspaces of $\operatorname{Spec}(R)$, which has been introduced and studied in [20] to classify, from a topological point of view, distinguished classes of Krull closure operations, namely the e.a.b. semistar operations and the stable semistar operations of finite type. In particular, we prove here that $\mathcal{S}(R)$ is a spectral retract of $\mathcal{X}(R)$ (Proposition 2.11) and we characterize when $\mathcal{S}(R)$ is canonically homeomorphic to $\mathcal{X}(R)$, both in general and when $\operatorname{Spec}(R)$ is a Noetherian space. In the general case, this happens under the purely algebraic condition that the radical of every finitely generated ideal of $R$ is the radical of a principal ideal (Theorem 2.13) and, in the Noetherian space case, when every prime ideal of $R$ is the radical of a principal ideal (Corollary 2.14). When $R$ is a Bézout domain, we prove that $\mathcal{S}(R)$ is canonically homeomorphic both to $\mathcal{X}(R)$ and to the space Overr $(R)$ of the overrings of $R$ endowed with the Zariski topology (Corollary 3.3). When $R$ is a Dedekind domain, $\mathcal{S}(R)$ is canonically homeomorphic to $\mathcal{X}(R)$ if and only if the ideal class group of $R$ is torsion (Remark 3.5). Each of the previous homeomorphisms can be interpreted as a topological "dual" statement to Hilbert's Nullstellensatz, providing a one-to-one correspondence, compatible with the natural orders, between inverse-closed subspaces of $\operatorname{Spec}(R)$ and semigroup primes of $R$.

In the final section, we compare the space $\mathcal{X}(R)$ with the space $\mathcal{S}(R(T))$ of semigroup primes of the Nagata ring $R(T)$ (where $T$ is an indeterminate over $R$ ). In particular, we provide a canonical spectral embedding $\mathcal{X}(R) \hookrightarrow \mathcal{S}(R(T))$ which makes $\mathcal{X}(R)$ a spectral retract of $\mathcal{S}(R(T))$ (Propositions 4.2 and 4.4).

In order to facilitate the reader, we recall next some preliminary notions and results that will be used in the present paper.

1.1. Spectral spaces. A topological space is spectral (after M. Hochster [36]) if it is homeomorphic to the prime spectrum of a (commutative) ring. While defined in algebraic terms, this concept admits a purely topological characterization: a topological space $X$ is spectral if and only if it is $\mathrm{T}_{0}$, quasi-compact, it admits a 
basis of open and quasi-compact subspaces that is closed under finite intersections, and every irreducible closed subset of $X$ has a (unique) generic point (i.e., it is the closure of a one-point set) [36. If $X$ and $Y$ are spectral spaces, a spectral map $f: X \rightarrow Y$ is a map such that $f^{-1}(U)$ is a quasi-compact open subspace of $X$, for each quasi-compact open subspace $U$ of $Y$; spectral maps are the morphisms in the category having the spectral spaces as objects.

It is well known that the prime spectrum of a commutative ring endowed with the Zariski topology is always $T_{0}$, but almost never Hausdorff (it is Hausdorff if and only if the ring has Krull dimension zero). Thus, many authors have considered a finer topology on the prime spectrum of a ring, known as the constructible topology [31. pages 337-339] or as the patch topology [36.

As in [55, we introduce the constructible topology by a Kuratowski closure operator: if $X$ is a spectral space, for each subset $Y$ of $X$, we set:

$$
\begin{aligned}
& \mathrm{Cl}^{\text {cons }}(Y):=\bigcap\{U \cup(X \backslash V) \mid U \text { and } V \text { open and quasi-compact in } X \text {, } \\
& U \cup(X \backslash V) \supseteq Y\} .
\end{aligned}
$$

We denote by $X^{\text {cons }}$ the set $X$, equipped with the constructible topology. For Noetherian topological spaces, the closed sets of this topology coincide with the "constructible sets" classically defined in [8]. It is well known that $X^{\text {cons }}$ is a spectral space and that the constructible topology is a refinement of the given topology which is always Hausdorff.

1.2. The inverse topology on a spectral space. Recall that the given topology on a spectral space $X$ induces a canonical partial order $\leq_{X}$, denoted simply by $\leq$ when no danger of confusion can arise, defined by $x \leq_{X} y$ if $y \in \mathrm{Cl}(\{x\})$, for $x, y \in$ $X$, where $\mathrm{Cl}(Y)$ denotes the closure of a subset $Y$ of $X$. The set $Y^{\text {gen }}:=\{x \in X \mid$ $y \in \mathrm{Cl}(\{x\})$, for some $y \in Y\}$ is called closure under generizations of $Y$. Similarly, using the opposite order, the set $Y^{\mathrm{sp}}:=\{x \in X \mid x \in \mathrm{Cl}(\{y\})$, for some $y \in Y\}$ is called closure under specializations of $Y$. We say that $Y$ is closed under generizations (respectively, closed under specializations) if $Y=Y^{\text {gen }}$ (respectively, $Y=$ $\left.Y^{\text {sp }}\right)$. It is straightforward that, for two elements $x, y$ in a spectral space $X$, we have:

$$
x \leq y \quad \Leftrightarrow \quad\{x\}^{\text {gen }} \subseteq\{y\}^{\text {gen }} \Leftrightarrow\{x\}^{\mathrm{sp}} \supseteq\{y\}^{\mathrm{sp}} .
$$

Given a spectral space $X$, Hochster [36, Proposition 8] introduced a new topology on $X$, that we call here the inverse topology, by defining a Kuratowski closure operator, for each subset $Y$ of $X$, as follows:

$$
\mathrm{Cl}^{\text {inv }}(Y):=\bigcap\{U \mid U \text { open and quasi-compact in } X, U \supseteq Y\} .
$$

If we denote by $X^{\text {inv }}$ the set $X$ equipped with the inverse topology, Hochster proved that $X^{\text {inv }}$ is still a spectral space and the partial order on $X$ induced by the inverse topology is the opposite order of that induced by the given topology on $X$ [36, Proposition 8]. In particular, the closure under generizations $\{x\}^{\text {gen }}$ of a singleton is closed in the inverse topology of $X$, since $\{x\}^{\text {gen }}=\bigcap\{U \mid U \subseteq$ $X$ quasi-compact and open, $x \in U\}$. On the other hand, it is trivial, by the definition, that the closure under specializations of a singleton $\{x\}^{\mathrm{sp}}$ is closed in the given topology of $X$, since $\{x\}^{\text {sp }}=\mathrm{Cl}(\{x\})$.

Finally, recall that, by [17, Remark 2.2], we have $\mathrm{Cl}^{\text {inv }}(Y)=\left(\mathrm{Cl}^{\text {cons }}(Y)\right)^{\text {gen }}$. It follows that each closed set in the inverse topology (called for short, inverse-closed) is closed under generizations and, from [17, Proposition 2.6], that a quasi-compact 
subspace $Y$ of $X$ closed for generizations is inverse-closed. On the other hand, the closure of a subset $Y$ in the given topology of $X, \mathrm{Cl}(Y)$, coincides with $\left(\mathrm{Cl}^{\text {cons }}(Y)\right)^{\text {sp }}$ [17, Remark 2.2].

1.3. The spectral space of the inverse-closed subspaces. Given a spectral space $X$, let $\mathcal{X}(X):=\left\{Y \subseteq X \mid Y \neq \emptyset, Y=\mathrm{Cl}^{\text {inv }}(Y)\right\}$, that is, $\mathcal{X}(X)$ is the set of all nonempty subset of $X$ that are closed in the inverse topology. If $X=\operatorname{Spec}(R)$ for some ring $R$, we write for short $\mathcal{X}(R)$ instead of $\mathcal{X}(\operatorname{Spec}(R))$.

We define a Zariski topology on $\mathcal{X}(X)$ by taking, as subbasis (in fact, a basis) of open sets, the sets of the form

$$
\mathcal{U}(\Omega):=\{Y \in \mathcal{X} \mid Y \subseteq \Omega\}
$$

where $\Omega$ varies among the quasi-compact open subspaces of $X$. Note that $\emptyset \neq \Omega \in$ $\mathcal{U}(\Omega)$, since a quasi-compact open subset $\Omega$ of $X$ is a closed in the inverse topology of $X$. Note also that, when $X=\operatorname{Spec}(R)$, for some ring $R$, a generic basic open set of the Zariski topology on $\mathcal{X}(R)$ is of the form

$$
\mathcal{U}(\mathrm{D}(J))=\{Y \in \mathcal{X}(R) \mid Y \subseteq \mathrm{D}(J)\}
$$

where $J$ is any finitely generated ideal of $R$, and, as usual,

$$
\mathrm{V}(J):=\{P \in \operatorname{Spec}(R) \mid J \subseteq P\} \quad \text { and } \quad \mathrm{D}(J):=\operatorname{Spec}(R) \backslash \mathrm{V}(J) .
$$

It was proved in [20, Theorem 3.2] that:

(1) the space $\mathcal{X}(X)$, endowed with the Zariski topology, is a spectral space;

(2) the canonical map $\varphi: X \hookrightarrow \mathcal{X}(X)$, defined by $\varphi(x):=\{x\}^{\text {gen }}$, for each $x \in X$, is a spectral embedding (and, in particular, an order-preserving embedding between ordered sets, with the ordering induced by the Zariski topologies).

1.4. Semistar operations. Let $D$ be an integral domain with quotient field $K$. Let $\overline{\boldsymbol{F}}(D)$ (respectively, $\boldsymbol{F}(D) ; \boldsymbol{f}(D)$ ) be the set of all nonzero $D$-submodules of $K$ (respectively, nonzero fractional ideals; nonzero finitely generated fractional ideals) of $D$ (thus, $\boldsymbol{f}(D) \subseteq \boldsymbol{F}(D) \subseteq \overline{\boldsymbol{F}}(D)$ ).

A mapping $\star: \overline{\boldsymbol{F}}(D) \longrightarrow \overline{\boldsymbol{F}}(D), E \mapsto E^{\star}$, is called a semistar operation of $D$ if, for all $z \in K, z \neq 0$ and for all $E, F \in \overline{\boldsymbol{F}}(D)$, the following properties hold: $\left(\star_{1}\right)(z E)^{\star}=z E^{\star} ;\left(\star_{2}\right) E \subseteq F \Rightarrow E^{\star} \subseteq F^{\star} ;\left(\star_{3}\right) E \subseteq E^{\star} ;$ and $\left(\star_{4}\right) E^{\star \star}:=\left(E^{\star}\right)^{\star}=$ $E^{\star}$. We denote the set of all semistar operations on $D$ by $\operatorname{SStar}(D)$.

Given a semistar operation $\star$ on $D$, a nonzero ideal $I$ of $D$ is called a quasi-ᄎ-ideal

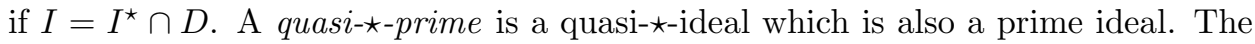
set of all quasi- $\star$-prime ideals of $D$ is denoted by $\mathrm{QSpec}^{\star}(D)$. The set of maximal elements in the set of proper quasi- $\star$-ideals of $D$ (ordered by set-theoretic inclusion) is denoted by $\mathrm{QMax}^{\star}(D)$ and it is a subset of QSpec $(D)$.

A semistar operation $\star$ is of finite type if, for every $E \in \overline{\boldsymbol{F}}(D)$,

$$
E^{\star}=\bigcup\left\{F^{\star} \mid F \subseteq E, F \in \boldsymbol{f}(D)\right\} .
$$

It is well known that if $\star$ is a semistar operation of finite type then $\mathrm{QMax}^{\star}(D)$ is nonempty [24, Lemma 2.3(1)].

For more details on semistar operations see, for instance, [14, [15], 32], 33, [42, and [46]; for the case of star operations see, for instance, [1], 2], 3], [13] and [28.

The set of all semistar operations of finite type is denoted by $\operatorname{SStar}_{f}(D)$. 
In [21, the set $\operatorname{SStar}(D)$ of all semistar operation was endowed with a topology (called the Zariski topology) having, as a subbasis of open sets, the sets of the type

$$
\mathrm{V}_{E}:=\left\{\star \in \operatorname{SStar}(D) \mid 1 \in E^{\star}\right\} \text {, where } E \text { is a nonzero } D \text {-submodule of } K \text {. }
$$

This topology makes $\operatorname{SStar}(D)$ into a quasi-compact $\mathrm{T}_{0}$ space, and $\operatorname{SStar}_{f}(D)$ into a spectral space.

1.5. Spectral semistar operations. Let $D$ be a domain and $Y \subseteq \operatorname{Spec}(D)$ be nonempty. The semistar operation $\mathbf{s}_{Y}$ is defined as the map such that

$$
E^{\mathbf{S}_{Y}}=\bigcap\left\{E D_{P} \mid P \in Y\right\} \quad \text { for every } E \in \overline{\boldsymbol{F}}(D) \text {. }
$$

The semistar operations on $D$ that can be written as $\mathbf{s}_{Y}$, for some $Y$, are called spectral; the set of all finite type spectral semistar operations, denoted by $\widetilde{\operatorname{SStar}}(D)$, is a spectral space [19, Theorem 4.6]. By [21, Corollary 4.4], $\mathrm{s}_{Y}$ is of finite type if and only if $Y$ is quasi-compact, as a subspace of $\operatorname{Spec}(D)$, endowed with the Zariski topology (see also [22] and [32]).

There is a canonical map

$$
\begin{aligned}
\widetilde{\Phi}: \operatorname{SStar}(D) & \longrightarrow \widetilde{\operatorname{SStar}}(D) \\
\star & \longmapsto \widetilde{\star},
\end{aligned}
$$

where $\widetilde{\star}$ is defined as the map such that, for every $E \in \overline{\boldsymbol{F}}(D)$,

$$
\begin{gathered}
E^{\widetilde{\star}}:=\bigcup\{(E: J) \mid J \text { nonzero finitely generated ideal of } D \\
\text { such that } \left.J^{\star}=D^{\star}\right\} .
\end{gathered}
$$

The map $\widetilde{\Phi}$ is a topological retraction [19, Proposition 4.3(2)]; in particular, $\star=\widetilde{\star}$ if and only if $\star$ is spectral and of finite type [22, Corollary 3.9(2)].

The space $\widetilde{\operatorname{SStar}}(D)$ can also be seen as a natural "extension" of $\operatorname{Spec}(D)$, since the canonical map s: $\operatorname{Spec}(D) \hookrightarrow \widetilde{\operatorname{SStar}}(D)$, defined by $P \mapsto \mathrm{s}_{\{P\}}$, is a topological embedding.

An alternative way to see the space $\widetilde{\operatorname{SStar}}(D)$ is through the space $\mathcal{X}(D)$ recalled in Section 1.3 By [20, Proposition 5.2], we have the following.

- The map $\mathbf{s}^{\sharp}: \mathcal{X}(D) \rightarrow \widetilde{\operatorname{SStar}}(D)$, defined by $Y \mapsto \mathbf{s}_{Y}$, and the map $\Delta$ : $\widetilde{\operatorname{SStar}}(D) \rightarrow \mathcal{X}(D)$, defined by $\star \mapsto \mathrm{QSpec}^{\star}(D)$, are homeomorphisms and are inverse of each other.

- If $\varphi: \operatorname{Spec}(D) \hookrightarrow \mathcal{X}(D)$ is the canonical embedding defined in 1.3(2), then $\mathrm{s} \sharp \varphi=\mathrm{s}$.

Remark 1.1. Let $\star$ be a semistar operation of finite type on the integral domain $D$. It is well known that $\mathrm{QMax}^{\star}(D)=\mathrm{QMax}^{\widetilde{\star}}(D)$ and $\widetilde{\star}=\mathrm{s}_{\mathrm{QSpec}}{ }^{\star}(D)=\mathrm{s}_{\mathrm{QMax}}{ }^{\star}(D)=$ $\mathrm{S}_{\mathrm{QMax}^{\tilde{*}}(D)}\left[24\right.$, Lemma 2.4 and Corollaries 2.7 and 3.5]. Moreover, since QSpec ${ }^{\widetilde{\star}}(D)$ is closed in the inverse topology of $\operatorname{Spec}(D)$ and the maps $\Delta, \mathrm{s}^{\sharp}$ are homeomorphisms (see above), it follows that $\mathrm{Cl}^{\mathrm{inv}}\left(\mathrm{QSpec}^{\star}(D)\right)=\mathrm{QSpec}^{\widetilde{\star}}(D)$. Therefore, by 21, Proposition 5.8], we also have

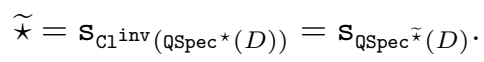


1.6. The set of overrings of an integral domain. Let Overr $(D)$ be the set of all overrings of $D$, endowed with the topology whose basic open sets are of the form $\mathrm{B}\left(x_{1}, x_{2}, \ldots, x_{r}\right):=0 \operatorname{verr}\left(D\left[x_{1}, x_{2}, \ldots, x_{n}\right]\right)$, for $x_{1}, x_{2}, \ldots, x_{n}$ varying in $K$ [58, Ch. VI, §17]. For recent investigations on topological spaces of overrings of an integral domain see, for instance, [18, [19, 44], 48, [49, 50].

It is known that:

(1) The topological space Overr $(D)$ is a spectral space [16, Proposition 3.5] and the map $\iota: \operatorname{Overr}(D) \hookrightarrow \operatorname{SStar}_{f}(D)$, defined by $\iota(T):=\wedge_{\{T\}}$, for each $T \in \operatorname{Overr}(D)$, is a topological embedding [21, Proposition 2.5].

(2) The map $\pi: \operatorname{SStar}_{f}(D) \rightarrow \operatorname{Overr}(D)$, defined by $\pi(\star):=D^{\star}$, for any $\star \in \operatorname{SStar}_{f}(D)$, is a topological retraction [18, Proposition 3.2].

\section{THE SPACE OF SEMIGRoup PRIMES}

Let $R$ be a ring. The purpose of the present section is to investigate a natural spectral extension of $\operatorname{Spec}(R)$ which is intermediate between $\operatorname{Spec}(R)$ and $\mathcal{X}(R)$, namely the embeding of the prime spectrum into the set of semigroup primes.

Using the terminology of [47, we recall the following definition:

Definition 2.1. A semigroup prime is a nonempty proper subset $\mathscr{Q}$ of a ring $R$ such that:

(a) for each $r \in R$ and for each $\pi \in \mathscr{Q}, r \pi \in \mathscr{Q}$;

(b) for all $\sigma, \tau \in R \backslash \mathscr{Q}, \sigma \tau \in R \backslash \mathscr{Q}$.

Obviously, every prime ideal of $R$ is also a semigroup prime of $R$. More generally, if $Y$ is a nonempty collection of prime ideals of $R$, then $\mathscr{P}(Y):=\bigcup\{P \in \operatorname{Spec}(R) \mid$ $P \in Y\}$ is a semigroup prime of $R$. A more precise result is given next.

Lemma 2.2. Let $\mathscr{Q}$ be a proper subset of a ring $R$. Then, $\mathscr{Q}$ is a semigroup prime of $R$ if and only if there exists a nonempty collection of prime ideals $Y$ of $R$ such that $\mathscr{Q}=\mathscr{P}(Y)$.

Proof. We just need to prove the "only if" part. For each semigroup prime $\mathscr{Q}$ of $R, R \backslash \mathscr{Q}$ is a multiplicatively closed subset of $R$ and it is also saturated, since if $\alpha \beta \in R \backslash \mathscr{Q}$ then, from (a) of the previous definition, it follows immediately that both $\alpha$ and $\beta$ belong to $R \backslash \mathscr{Q}$. Since a saturated multiplicatively closed set is the complement of the union of prime ideals [39, Theorem 2], if $Y$ is a nonempty set of prime ideals of $R$ such that $R \backslash \mathscr{P}(Y)$ coincides with the saturated multiplicatively closed set $R \backslash \mathscr{Q}$, then $\mathscr{Q}=\mathscr{P}(Y)$.

Let $\mathcal{S}(R):=\{\mathscr{Q} \mid \mathscr{Q}$ is a semigroup prime of $R\}$. As in [47, (2.3)], the set $\mathcal{S}(R)$ can be endowed with a hull-kernel topology, by taking as a basis for the open sets the subsets

$$
\boldsymbol{U}\left(x_{1}, x_{2}, \ldots, x_{n}\right):=\left\{\mathscr{Q} \mid x_{i} \notin \mathscr{Q} \text { for some } i, 1 \leq i \leq n\right\},
$$

where $x_{1}, x_{2}, \ldots, x_{n} \in R$.

Proposition 2.3. Let $R$ be a ring.

(1) The set $\mathcal{S}(R)$ of semigroup primes of $R$ with the hull-kernel topology is a spectral space.

(2) The collection of sets $\{\boldsymbol{U}(x) \mid x \in R\}$ is a basis of open and quasi-compact subspaces of $\mathcal{S}(R)$. 
(3) The set theoretic inclusion $i: \operatorname{Spec}(R) \hookrightarrow \mathcal{S}(R)$ is a spectral embedding.

Proof. (1) Since $R \backslash \mathscr{Q}$ is a saturated multiplicative set of $R$ for each $\mathscr{Q} \in \mathcal{S}(R)$, then $\boldsymbol{U}(x y)=\boldsymbol{U}(x) \cap \boldsymbol{U}(y)$ for each pair $x, y \in R$. By definition, it follows easily that a basis of open sets for $\mathcal{S}(R)$ is given by $\{\boldsymbol{U}(x) \mid x \in R\}$.

By [16, Corollary 3.3], to show that $\mathcal{S}(R)$ is a spectral space it suffices to show that, for any ultrafilter $\mathscr{U}$ on $\mathcal{S}(R)$, the set

$$
\{\mathscr{Q} \in \mathcal{S}(R) \mid \forall x \in R, \mathscr{Q} \in \boldsymbol{U}(x) \Leftrightarrow \boldsymbol{U}(x) \in \mathscr{U}\}
$$

is nonempty. Set $\mathscr{Q}_{\mathscr{U}}:=\{r \in R \mid \mathcal{S}(R) \backslash \boldsymbol{U}(r) \in \mathscr{U}\}$. An easy argument shows that $\mathscr{Q}_{\mathscr{U}}$ is a semigroup prime of $R$. Moreover, by definition, for each $x \in R$, $\mathscr{Q}_{\mathscr{U}} \in \boldsymbol{U}(x)$ if and only if $\boldsymbol{U}(x) \in \mathscr{U}$.

(2) By [16, Propositions 2.11, 3.1(3,b) and 3.2], the sets $\boldsymbol{U}(x)$ are clopen, with respect to the constructible topology of $\mathcal{S}(R)$ and, a fortiori, they are quasi-compact with respect to the hull-kernel topology.

(3) The conclusion follows from the fact that the hull-kernel topology of $\mathcal{S}(R)$ induces the Zariski topology on $\operatorname{Spec}(R)$, since $i^{-1}(\boldsymbol{U}(x))=\boldsymbol{U}(x) \cap \operatorname{Spec}(R)=\mathrm{D}(x)$ and from the fact that $i(\mathrm{D}(x))=\boldsymbol{U}(x) \cap i(\operatorname{Spec}(R))$, for each $x \in R$.

Remark 2.4. Let $S$ be a semigroup. A prime ideal of $S$ is a nonempty proper subset $I \subseteq S$ such that $x s \in I$ for every $x \in I, s \in S$ and such that $s t \in S \backslash I$ for every $s, t \in S \backslash I$ (see, for example, [30, 40). Under this terminology, a prime semigroup of a ring $R$ is just a prime ideal of the multiplicative $\operatorname{semigroup}(R, \cdot)$.

The topology we introduced above in the case of prime semigroups of a ring can be extended naturally to the set $\mathcal{S}(S)$ of the prime ideals of the semigroup $S$; likewise, the proof of Proposition 2.3(1) can be transferred verbatim to the case of semigroups, showing the slightly more general result that $\mathcal{S}(S)$ is a spectral space.

Remark 2.5. The subspace $\operatorname{Spec}(R)$ of $\mathcal{S}(R)$ is dense in $\mathcal{S}(R)$. In fact, the closure of $\operatorname{Spec}(R)$ is the set of all $\mathscr{Q} \in \mathcal{S}(R)$ containing the nilradical of $R$, which is $\mathcal{S}(R)$ (since each $\mathscr{Q}$ contains at least one prime $P \in \operatorname{Spec}(R)$ ).

Following [31, Définition (2.6.3)], recall that a subset $X_{0}$ of a topological space $X$ is said to be very dense in $X$ if, for any open sets $U, V \subseteq X$, the equality $U \cap X_{0}=V \cap X_{0}$ implies $U=V$, that is, in our setting, if the map $\boldsymbol{U} \mapsto \boldsymbol{U} \cap \operatorname{Spec}(R)$, from the open subsets of $\mathcal{S}(R)$ to the open subsets of $\operatorname{Spec}(R)$, is injective. Under this terminology, $\operatorname{Spec}(R)$ is not very dense in $\mathcal{S}(R)$. For instance, consider a 1dimensional Bézout domain $D$ with exactly two maximal ideals, say $M$ and $N$. Then, $\mathcal{S}(D)$ has a maximal element (namely $M \cup N$ ) that is a closed point but does not belong to $\operatorname{Spec}(D)$.

Given a ring homomorphism $f: R_{1} \rightarrow R_{2}$, we can canonically associate to $f$ a map

$$
\begin{aligned}
\mathcal{S}(f): \mathcal{S}\left(R_{2}\right) & \longrightarrow \mathcal{S}\left(R_{1}\right) \\
\mathscr{Q} & \longmapsto f^{-1}(\mathscr{Q}) .
\end{aligned}
$$

We investigate next the properties of this map.

Proposition 2.6. Let $f: R_{1} \rightarrow R_{2}$ be a ring homomorphism, let $\mathcal{S}(f)$ be the map defined above and let $f^{a}: \operatorname{Spec}\left(R_{2}\right) \rightarrow \operatorname{Spec}\left(R_{1}\right)$ be the continuous map canonically associated to $f$. Assume that $\mathcal{S}\left(R_{1}\right)$ and $\mathcal{S}\left(R_{2}\right)$ are endowed with the hull-kernel topology. Then: 
(1) $\mathcal{S}(f)$ is well-defined, (continuous) and spectral;

(2) if $i_{k}: \operatorname{Spec}\left(R_{k}\right) \longrightarrow \mathcal{S}\left(R_{k}\right)$ is the set-theoretic inclusion $(k=1,2)$, then $\mathcal{S}(f) \circ i_{2}=i_{1} \circ f^{a}$

(3) the assignment $R \mapsto \mathcal{S}(R), f \mapsto \mathcal{S}(f)$, is a functor from the category of rings to the category of spectral spaces.

Proof. (1) Let $\mathscr{Q}$ be a semigroup prime of $R_{2}$, let $r \in R_{1}$ and $\pi \in f^{-1}(\mathscr{Q})$. Then, $f(\pi r)=f(\pi) f(r) \in f(r) \mathscr{Q} \subseteq \mathscr{Q}$, so that $r \pi \in f^{-1}(\mathscr{Q})$; moreover, if $\sigma, \tau \notin f^{-1}(\mathscr{Q})$, then $f(\sigma), f(\tau) \notin \mathscr{Q}$ and thus $f(\sigma) f(\tau) \notin \mathscr{Q}$, that is, $\sigma \tau \notin f^{-1}(\mathscr{Q})$. Hence, $\mathcal{S}(f)$ is well-defined. Moreover, $\mathcal{S}(f)^{-1}(\boldsymbol{U}(x))=\boldsymbol{U}(f(x))$ for each $x \in R_{1}$, and thus $\mathcal{S}(f)$ is continuous. By the last part of Proposition 2.3 (1), the collection $\{\boldsymbol{U}(y) \mid y \in A\}$ is a basis of quasi-compact subsets of $\mathcal{S}(A)$, for any ring $A$. Thus, the previous reasoning implies that $\mathcal{S}(f)$ is a spectral map.

(2) is straightforward.

(3) follows from the previous points and the fact that, given two ring homomorphisms $f: R_{1} \rightarrow R_{2}$ and $g: R_{2} \rightarrow R_{3}, \mathcal{S}(g \circ f)=\mathcal{S}(f) \circ \mathcal{S}(g)$, which is a direct consequence of the definitions.

We now start the study of the relationship between the spectral spaces $\mathcal{S}(R)$ and $\mathcal{X}(R)$.

Proposition 2.7. Let $R$ be a ring.

(1) For each $\mathscr{Q} \in \mathcal{S}(R)$, set $\Sigma_{\mathscr{Q}}:=R \backslash \mathscr{Q}$ and $R_{\mathscr{Q}}:=\Sigma_{\mathscr{Q}}^{-1} R$. The map

$$
\begin{aligned}
j: \mathcal{S}(R) & \longrightarrow \mathcal{X}(R) \\
\mathscr{Q} & \longmapsto \lambda^{a}\left(\operatorname{Spec}\left(R_{\mathscr{Q}}\right)\right),
\end{aligned}
$$

where $\lambda^{a}: \operatorname{Spec}\left(R_{\mathscr{Q}}\right) \rightarrow \operatorname{Spec}(R)$ is the spectral map associated to the localization homomorphism $\lambda: R \rightarrow R_{\mathscr{Q}}$, is a topological embedding. Moreover, $j(\mathscr{Q})=\{P \in \operatorname{Spec}(R) \mid P \subseteq \mathscr{Q}\}$, for each $\mathscr{Q} \in \mathcal{S}(R)$.

(2) The canonical spectral embedding $\varphi: \operatorname{Spec}(R) \hookrightarrow \mathcal{X}(R)$ [20, Theorem $3.3(2)]$ coincides with $j \circ i$.

Proof. (1) The map $j$ is clearly injective. In order to prove that $j$ is continuous we have to verify that, given a nonzero finitely generated ideal $J$ of $R$, then

$$
H:=j^{-1}(\mathcal{U}(\mathrm{D}(J)))=\{\mathscr{Q} \in \mathcal{S}(R) \mid j(\mathscr{Q}) \subseteq \mathrm{D}(J)\}
$$

is open in $\mathcal{S}(R)$. Take a point $\mathscr{Q} \in H$ and assume that $J \subseteq \mathscr{Q}$. Then $J$ is disjoint from $\Sigma_{\mathscr{Q}}$, and thus there exists a prime ideal $P$ of $R$ disjoint from $\Sigma_{\mathscr{Q}}$ and such that $J \subseteq P$. On the other hand, keeping in mind that $\mathscr{Q} \in H$ and $P \cap \Sigma_{\mathscr{Q}}=\emptyset$, we have $P \in j(\mathscr{Q}) \subseteq \mathrm{D}(J)$, contradiction. This shows that $J \nsubseteq \mathscr{Q}$, and thus there exists an element $x \in J \backslash \mathscr{Q}$. It follows that $\mathscr{Q} \in \boldsymbol{U}(x)$ and, moreover, $\boldsymbol{U}(x) \subseteq H$. Since $\{\boldsymbol{U}(x) \mid x \in R\}$ is a basis of open sets for $\mathcal{S}(R)$, it follows that $H$ is open and $j$ is continuous. Now, the fact that $j$ is a topological embedding follows immediately from the equality $j(\boldsymbol{U}(x))=j(\mathcal{S}(R)) \cap \mathcal{U}(\mathrm{D}(x))$ that holds for each $x \in R$.

For the last statement, we have $P \in \lambda^{a}\left(\operatorname{Spec}\left(R_{\mathscr{Q}}\right)\right)$ if and only if $P \cap \Sigma_{\mathscr{Q}}=\emptyset$, i.e., if and only if $P \subseteq \mathscr{Q}$.

(2) is a straightforward consequence of the definitions.

Proposition 2.8. Let $f: R_{1} \rightarrow R_{2}$ be a ring homomorphism, $f^{a}: \operatorname{Spec}\left(R_{2}\right) \rightarrow$ $\operatorname{Spec}\left(R_{1}\right)$ the associated map of spectra, $\mathcal{S}(f)$ the map defined in (11), $\mathcal{X}\left(f^{a}\right)$ : $\mathcal{X}\left(R_{2}\right) \rightarrow \mathcal{X}\left(R_{1}\right)$ the spectral map defined in [20, Proposition 4.1] and let $i_{k}$ : 
$\operatorname{Spec}\left(R_{k}\right) \rightarrow \mathcal{S}\left(R_{k}\right)$ (respectively, $j_{k}: \mathcal{S}\left(R_{k}\right) \rightarrow \mathcal{X}\left(R_{k}\right)$ ) the spectral embedding defined in Proposition 2.3 (respectively, Proposition 2.7), for $k=1,2$. Then, the diagram:

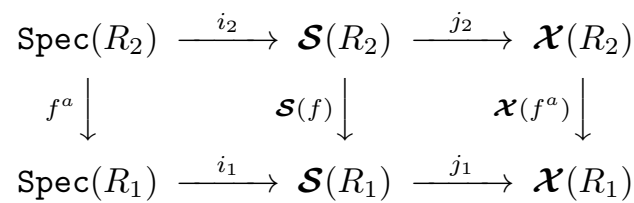

commutes.

Proof. The left square of (2) commutes by Proposition 2.6(2).

Let now $\mathscr{Q} \in \mathcal{S}\left(R_{2}\right)$. Then, using Proposition 2.7(1),

$$
j_{1} \circ \mathcal{S}(f)(\mathscr{Q})=j_{1}\left(f^{-1}(\mathscr{Q})\right)=\left\{P \mid P \subseteq f^{-1}(\mathscr{Q})\right\},
$$

while

$$
\begin{aligned}
\mathcal{X}\left(f^{a}\right) \circ j_{2}(\mathscr{Q}) & =\mathcal{X}\left(f^{a}\right)(\{P \mid P \subseteq \mathscr{Q}\}) \\
& =\left(f^{a}(\{P \mid P \subseteq \mathscr{Q}\})\right)^{\text {gen }} \\
& =\left(\left\{f^{-1}(P) \mid P \subseteq \mathscr{Q}\right\}\right)^{\text {gen }} .
\end{aligned}
$$

Let $Q \in \operatorname{Spec}\left(R_{1}\right)$. If $Q \in \mathcal{X}\left(f^{a}\right) \circ j_{2}(\mathscr{Q})$, then $Q \subseteq f^{-1}(P)$ for some $P \subseteq \mathscr{Q}$; hence, $Q \subseteq f^{-1}(\mathscr{Q})$ and $Q \in j_{1} \circ \mathcal{S}(f)(\mathscr{Q})$.

Conversely, suppose $Q \in j_{1} \circ \mathcal{S}(f)(\mathscr{Q})$, then $Q \subseteq f^{-1}(\mathscr{Q})$. Therefore, $f(Q) \subseteq \mathscr{Q}$ and so $f(Q) R_{2} \cap \Sigma_{\mathscr{Q}}=\emptyset$, where $\Sigma_{\mathscr{Q}}:=R_{2} \backslash \mathscr{Q}$. It follows that $f(Q) R_{2}$ extends to a proper ideal of $\Sigma_{\mathscr{Q}}^{-1} R_{2}$, and in particular there is a prime ideal $P$ of $R_{2}$ such that $f(Q) \subseteq P$ and $\Sigma_{\mathscr{Q}}^{-1} P \neq \Sigma_{\mathscr{Q}}^{-1} R_{2}$. Therefore, $P \subseteq \mathscr{Q}$. It follows that $Q \subseteq$ $f^{-1}(f(Q)) \subseteq f^{-1}(P)\left(\subseteq f^{-1}(\mathscr{Q})\right)$, and so $Q \in \mathcal{X}\left(f^{a}\right) \circ j_{2}(\mathscr{Q})$. Therefore, also the right square of (2) commutes.

It is obvious that, if $f$ is an isomorphism, $\mathcal{S}(f)$ is a homeomorphism. The converse does not hold; for example, if $R_{1} \subset R_{2}$ is a proper integral extension of one-dimensional local domains, then $\mathcal{S}(f)$ (like $f^{a}$ and $\mathcal{X}\left(f^{a}\right)$ ) is a homeomorphism, but $f$ is not an isomorphism. More generally, we have:

Corollary 2.9. Let $f: R_{1} \rightarrow R_{2}$ be a ring homomorphism, and let $f^{a}: \operatorname{Spec}\left(R_{2}\right) \rightarrow$ $\operatorname{Spec}\left(R_{1}\right)$ be the associated spectral map. If $f^{a}$ is a topological embedding (respectively, a homeomorphism) then so is $\mathcal{S}(f)$.

Proof. If $f^{a}$ is a topological embedding then, by [20, Proposition 4.4(1)], so is $\mathcal{X}\left(f^{a}\right)$, and thus also $\mathcal{X}\left(f^{a}\right) \circ j_{2}$ is a topological embedding. By Proposition 2.8, it follows that $j_{1} \circ \mathcal{S}(f)$ is a topological embedding, and thus so is $\mathcal{S}(f)$.

If $f^{a}$ is a homeomorphism, then by the previous paragraph $\mathcal{S}(f)$ is a topological embedding. Let $\mathscr{Q} \in \mathcal{S}\left(R_{1}\right)$, and let $\mathscr{L}:=\bigcup\left\{\operatorname{rad}\left(f(P) R_{2}\right) \mid P \subseteq \mathcal{Q}\right\}$. Since $f^{a}$ is a homeomorphism, $\operatorname{rad}\left(f(P) R_{2}\right)$ is a prime ideal of $R_{2}$ (since the irreducible closed $\mathrm{V}(P)$ subspace of $\operatorname{Spec}\left(R_{1}\right)$ is homeomorphic to $\mathrm{V}\left(\operatorname{rad}\left(f(P) R_{2}\right)\right)$ in $\left.\operatorname{Spec}\left(R_{2}\right)\right)$, and so $\mathscr{L}$ is a prime semigroup. We claim that $\mathcal{S}(f)(\mathscr{L})=\mathscr{Q}$. Clearly if $q \in \mathscr{Q}$ then $f(q) \in \mathscr{L}$, and $q \in f^{-1}(\mathscr{L})=\mathcal{S}(f)(\mathscr{L})$. Conversely, if $q \in \mathcal{S}(f)(\mathscr{L})$, then $f(q)^{n} \in$ $f(P) R_{2}$ for some $P \subseteq \mathscr{Q}$ and for some $n \geq 1$. Hence $q^{n} \in f^{-1}\left(f(P) R_{2}\right)=P$, the last equality coming from the bijectivity of $f^{a}$. Thus, $q \in P \subseteq \mathscr{Q}$. Therefore, $\mathcal{S}(f)$ is surjective, and thus a homeomorphism.

Remark 2.10. Despite the similarity between the properties enjoyed by $\mathcal{X}(R)$ and $\mathcal{S}(R)$, there is however a significant difference: while $\mathcal{X}(R)$ is a purely topological 
construction (depending only on the topology of $\operatorname{Spec}(R)$, see [20, Theorem 3.2 and Corollary 4.9]), $\mathcal{S}(R)$ depends also on the algebraic properties of $R$. In particular, $\mathcal{S}(R)$, in contrast with $\mathcal{X}(R)$ [20, Theorem 4.5] cannot be obtained from $\operatorname{Spec}(R)$ alone through a universal property. We provide now an example of this fact, and another example will be given later (Example 3.4).

Unlike in the case of $\mathcal{X}(R)$ [20, Proposition 4.4], the image of $\operatorname{Spec}(R)$ in $\mathcal{S}(R)$ cannot be determined uniquely by topological means. For example, let $R$ be a unique factorization domain, and let $\mathcal{P}(R)$ be the set of equivalence classes of prime elements of $R$ modulo multiplication by units. Any prime semigroup in $\mathcal{S}(R)$ is uniquely determined by the prime elements that it contains, and thus there is a bijective correspondence between $\mathcal{S}(R)$ and the power set $\mathscr{B}:=\mathscr{B}(\mathcal{P}(R))$ of $\mathcal{P}(R)$, which becomes a homeomorphism if we take, as a subbasis for $\mathscr{B}$, the family of the subsets of $\mathscr{B}$ of the form $\boldsymbol{V}(p):=\{B \in \mathscr{B} \mid p \notin B\}$, as $p$ runs in $\mathcal{P}(R)$. In particular, the topology of $\mathcal{S}(R)$ depends uniquely on the cardinality of $\mathcal{P}(R)$, and thus it does not depend on other properties of $R$ or $\operatorname{Spec}(R)$ : for example, it does not depend on the dimension of $R$. Hence, by cardinality reasons, there exists a homeomorphism $\mathcal{S}(\mathbb{Z}) \simeq \mathcal{S}(\mathbb{Z}[X])$, but $j(\operatorname{Spec}(\mathbb{Z}))$ and $j(\operatorname{Spec}(\mathbb{Z}[X]))$ are not homeomorphic, and so they do not correspond under any homeomorphism between $\mathcal{S}(\mathbb{Z})$ and $\mathcal{S}(\mathbb{Z}[X])$.

We prove next that the spectral space $\mathcal{S}(R)$ is a retract of the spectral space $\mathcal{X}(R)$.

Proposition 2.11. Let $R$ be a ring, $j: \mathcal{S}(R) \rightarrow \mathcal{X}(R)$ the canonical embedding defined in Proposition 2.7(1) and let $\mathscr{P}: \mathcal{X}(R) \rightarrow \mathcal{S}(R)$ be the map defined by setting $\mathscr{P}(Y):=\bigcup\{P \mid P \in Y\}$ for each $Y \in \mathcal{X}(R)$. Then:

(1) $\mathscr{P}$ is surjective and spectral;

(2) $\mathscr{P} \circ j$ is the identity on $\mathcal{S}(R)$;

(3) for every $Y \in \mathcal{X}(R),(j \circ \mathscr{P})(Y)=\bigcap\{\mathrm{D}(a) \mid Y \subseteq \mathrm{D}(a)\}$.

Proof. (1) and (2). Let $\boldsymbol{U}(x)$ be a basic open set of $\mathcal{S}(R)$, with $x \in R$. Then,

$$
\begin{aligned}
\mathscr{P}^{-1}(\boldsymbol{U}(x))= & \{Y \in \mathcal{X}(R) \mid \mathscr{P}(Y) \in \boldsymbol{U}(x)\}= \\
& =\{Y \in \mathcal{X}(R) \mid x \notin \mathscr{P}(Y)\} \\
& =\{Y \in \mathcal{X}(R) \mid x \notin \bigcup\{P \mid P \in Y\}\} \\
& =\{Y \in \mathcal{X}(R) \mid x \notin P \text { for every } P \in Y\}= \\
& =\{Y \in \mathcal{X}(R) \mid Y \subseteq \mathrm{D}(x)\}=\mathcal{U}(\mathrm{D}(x))
\end{aligned}
$$

which is a basic quasi-compact open set of $\mathcal{X}(R)$. Hence, $\mathscr{P}$ is (continuous and) spectral.

The fact that $\mathscr{P} \circ j$ is the identity on $\mathcal{S}(R)$ follows directly from Lemma 2.2 and Proposition 2.7 (1), and in particular it implies that $\mathscr{P}$ is surjective.

(3) Let $Y \in \mathcal{X}(R)$. If $Y \subseteq \mathrm{D}(a)$, then $a \notin P$ for every $P \in Y$, and thus $a \notin \bigcup\{P \mid P \in Y\}=\mathscr{P}(Y)$. Hence, if $Q \in(j \circ \mathscr{P})(Y)$ then $a \notin Q$ and so $Q \in \mathrm{D}(a)$. Conversely, suppose $Q$ belongs to the given intersection. If $Q \notin(j \circ \mathscr{P})(Y)$, then an element $q \in Q \backslash \mathscr{P}(Y)$ would exist. But this would imply $Y \subseteq \mathrm{D}(q)$ while $Q \notin \mathrm{D}(q)$, which is absurd.

Remark 2.12. As we observed at the beginning of the present section, we can define $\mathscr{P}(Y):=\{P \mid P \in Y\}$ for each nonempty subset $Y$ of $\operatorname{Spec}(R)$. In this case, we can show that if $Y_{1}, Y_{2} \subseteq \operatorname{Spec}(R)$ and if $\mathrm{Cl}^{\text {inv }}\left(Y_{1}\right) \subseteq \mathrm{Cl}^{\text {inv }}\left(Y_{2}\right)$ then 
$\mathscr{P}\left(Y_{1}\right) \subseteq \mathscr{P}\left(Y_{2}\right)$. In particular, if $\mathrm{Cl}^{\text {inv }}\left(Y_{1}\right)=\mathrm{Cl}^{\text {inv }}\left(Y_{2}\right)$, then $\mathscr{P}\left(Y_{1}\right)=\mathscr{P}\left(Y_{2}\right)$, hence $\mathscr{P}(Y)=\mathscr{P}\left(\mathrm{Cl}^{\text {inv }}(Y)\right)$ for each nonempty subset $Y$ of $\operatorname{Spec}(R)$.

As a matter of fact, let $x \in R$ be such that $x \in \mathscr{P}\left(Y_{1}\right) \backslash \mathscr{P}\left(Y_{2}\right)$. Then $\mathrm{D}(x)$ contains $Y_{2}$, and it is a closed set, with respect to the inverse topology of $\operatorname{Spec}(R)$. Thus, by assumption, $\mathrm{D}(x) \supseteq \mathrm{Cl}^{\text {inv }}\left(Y_{2}\right) \supseteq \mathrm{Cl}^{\text {inv }}\left(Y_{1}\right) \supseteq Y_{1}$. On the other hand, since $x \in \mathscr{P}\left(Y_{1}\right)$, there exist a prime ideal $P \in Y_{1}$ such that $x \in P$, and hence $Y_{1} \nsubseteq \mathbb{\mathrm { D }}(x)$, which is a contradiction.

In the next result, we characterize when the canonical embedding $\mathcal{S}(R) \hookrightarrow \mathcal{X}(R)$ is a homeomorphism and, as a consequence, we deduce that, in general, there are rings $R$ and inverse-closed subspaces $Y$ of $\operatorname{Spec}(R)$ such that $Y \subsetneq(j \circ \mathscr{P})(Y)$.

Theorem 2.13. Let $R$ be a ring. The following statements are equivalent.

(i) The canonical embedding $j: \mathcal{S}(R) \hookrightarrow \mathcal{X}(R)$ (defined in Proposition 2.7(1)) is a homeomorphism.

(ii) The radical of every finitely generated ideal of $R$ is the radical of a principal ideal.

(iii) If $I$ is a finitely generated ideal of $R$ and $I \subseteq \mathscr{Q}:=\bigcup\left\{Q_{\lambda} \mid \lambda \in \Lambda\right\} \in \mathcal{S}(R)$ (where $Q_{\lambda} \in \operatorname{Spec}(R)$ for each $\lambda$ ), then $I \subseteq Q_{\lambda}$ for some $\lambda \in \Lambda$.

(iv) A basis for the open sets for the Zariski topology of $\mathcal{X}(R)$ is given by the collection $\{\mathcal{U}(D(x)) \mid x \in R\}$.

Proof. (i) $\Rightarrow$ (ii). By Proposition 2.7(1), $j$ is a homeomorphism if and only if it is surjective. Suppose $j$ is a homeomorphism, and suppose there is a nonzero finitely generated ideal $I$ such that $\operatorname{rad}(I) \neq \operatorname{rad}(a R)$ for every $a \in R$. Consider $Y:=\mathrm{D}(I)$ : then, $Y$ is open and quasi-compact in the Zariski topology, and thus it is a closed set in the inverse topology. Since $j$ is surjective, there is a prime semigroup $\mathscr{Q}$ such that $Y=j(\mathscr{Q})$. Set $\Sigma_{\mathscr{Q}}:=R \backslash \mathscr{Q}$ and $R_{\mathscr{Q}}:=\Sigma_{\mathscr{Q}}^{-1} R$. Suppose $I \subseteq \mathscr{Q}$ : then, $I R_{\mathscr{Q}} \neq R_{\mathscr{Q}}$, so that there is a prime ideal $P$ such that $I \subseteq P$ and $P R_{\mathscr{Q}} \neq R_{\mathscr{Q}}$. Therefore, $P \notin \mathrm{D}(I)=Y$. On the other hand, $P \subseteq \mathscr{Q}$, and thus $P \in j(\mathscr{Q})=Y$ : a contradiction. Henceforth, $I \nsubseteq \mathscr{Q}$, i.e., there exists an element $s \in I \cap \Sigma_{\mathscr{Q}}$. However, since the radical of the ideal $s R$ cannot be equal to $\operatorname{rad}(I)$, and $s R \subseteq I$, there is a prime ideal $Q$ containing $s R$ but not $I$. Hence, $Q \in Y$, while $Q R_{\mathscr{Q}}=R_{\mathscr{Q}}$, and thus $Q \notin j(\mathscr{Q})$. Again, this conflicts with the assumptions, and so we conclude that $Y$ is not in the image of $j$.

(ii) $\Rightarrow$ (iii). Let $I$ be a nonzero finitely generated ideal of $R$ and assume that $I \subseteq \mathscr{Q}$. By hypothesis, $\operatorname{rad}(I)=\operatorname{rad}(s R)$ for some $s$, and we can suppose $s \in I$. Since $I \subseteq \bigcup\left\{Q_{\lambda} \mid \lambda \in \Lambda\right\}$, then $s \in Q_{\lambda}$ for some $\lambda \in \Lambda$ and, hence, $I \subseteq \operatorname{rad}(I)=$ $\operatorname{rad}(s R) \subseteq Q_{\lambda}$.

(iii) $\Rightarrow$ (i). Let $Y \in \mathcal{X}(R)$, and let $\mathscr{P}(Y)=\bigcup\left\{Q_{\lambda} \mid \lambda \in \Lambda\right\} \in \mathcal{S}(R)$. We claim that $j(\mathscr{P}(Y))=Y$. Clearly, $Y \subseteq j(\mathscr{P}(Y)$ ) (Proposition 2.11(3)). On the other hand, suppose $P \in j(\mathscr{P}(Y)) \backslash Y$. Then, since $Y=\mathrm{Cl}^{\text {inv }}(Y)$, there is a basic closed set $\Omega=\mathrm{D}(I)$ of the inverse topology on $\operatorname{Spec}(R)$, such that $Y \subseteq \Omega$ but $P \notin \Omega$. Since $\Omega$ is quasi-compact in the Zariski topology, we can suppose $I$ finitely generated. The fact that $P \notin \mathrm{D}(I)$ implies $I \subseteq P$. On the other hand, $P \in j(\mathscr{P}(Y))$, hence $P \subseteq \bigcup\{Q \mid Q \in Y\}$. Therefore, by hypothesis, there is a $\bar{Q} \in Y(\subseteq \Omega)$ such that $I \subseteq \bar{Q}$; but this would imply $\bar{Q} \notin \mathrm{D}(I)$, which is absurd. Hence, $Y$ is in the image of $j$, and so $j$ is surjective.

Clearly, (ii) $\Rightarrow$ (iv) since a basis for the open sets of $\mathcal{X}(R)$ is given by $\mathcal{U}(\mathrm{D}(J))$ for $J$ varying among the finitely generated ideals of $R$. Conversely, let $J$ be a nonzero 
finitely generated ideal of $R$. Since $\mathrm{D}(J) \in \mathcal{U}(\mathrm{D}(J))$, by assumption there is an element $x \in R$ such that $\mathrm{D}(J) \in \mathcal{U}(\mathrm{D}(x)) \subseteq \mathcal{U}(\mathrm{D}(J))$, that is, $\mathrm{D}(x)=\mathrm{D}(J)$ and, in other words, $\operatorname{rad}(x R)=\operatorname{rad}(J)$.

An example where the previous theorem can be applied is when $R$ contains an uncountable field but its spectrum is only countable [54, Proposition 2.5].

In case $\operatorname{Spec}(R)$ is a Noetherian space, we have the following.

Corollary 2.14. Let $R$ be a ring. The following statements are equivalent.

(i) The canonical embedding $j: \mathcal{S}(R) \rightarrow \mathcal{X}(R)$ is a homeomorphism and $\operatorname{Spec}(R)$ is a Noetherian space.

(ii) Every prime ideal of $R$ is the radical of a principal ideal.

(iii) If $I$ is an ideal of $R$ and $I \subseteq \mathscr{Q}:=\bigcup\left\{Q_{\lambda} \mid \lambda \in \Lambda\right\} \in \mathcal{S}(R)$ (where $Q_{\lambda} \in \operatorname{Spec}(R)$ for each $\left.\lambda\right)$, then $I \subseteq Q_{\lambda}$ for some $\lambda \in \Lambda$.

(iv) If $P$ is a prime ideal of $R$ and $P \subseteq \mathscr{Q}:=\bigcup\left\{Q_{\lambda} \mid \lambda \in \Lambda\right\} \in \mathcal{S}(R)$ (where $Q_{\lambda} \in \operatorname{Spec}(R)$ for each $\lambda$ ), then $P \subseteq Q_{\lambda}$ for some $\lambda \in \Lambda$.

Proof. The equivalence of (i) and (ii) follows from the previous theorem, since $\operatorname{Spec}(R)$ is Noetherian if and only if every radical ideal is the radical of a finitely generated ideal (see for instance [43] or [23, Theorem 3.1.11]). The equivalences (ii) $\Leftrightarrow$ (iii) $\Leftrightarrow$ (iv) are due to W.W. Smith [56].

Remark 2.15. Rings verifying property (iii) of the previous corollary has been called compactly packed in [53].

Remark 2.16. It is well known that the rings verifying the equivalent conditions (ii)-(iv) of the previous corollary have Noetherian spectrum. On the other hand, Theorem 2.13 implies that $j$ is surjective for any Bézout domain and there are examples of Bézout domains (or, even, valuation domains) $R$ such that $\operatorname{Spec}(R)$ is not Noetherian. Therefore, for an arbitrary ring $R$, conditions (ii) $\Leftrightarrow$ (iii) $\Leftrightarrow$ (iv) of the previous corollary do not provide a characterization of when $j: \mathcal{S}(R) \longrightarrow \mathcal{X}(R)$ is a homeomorphism. In other words, the property that $j: \mathcal{S}(R) \rightarrow \mathcal{X}(R)$ is a homeomorphism does not depend only on the topology of the spectrum of $R$, but also on the algebraic structure of $R$.

Remark 2.17. (a) Let $R$ be a ring. If $\mathcal{T}:=\left\{\mathscr{Q}_{\alpha} \mid \alpha \in A\right\}$ is a nonempty subset of $\mathcal{S}(R)$, then $\bigcup\left\{\mathscr{Q}_{\alpha} \mid \alpha \in A\right\}$ is a prime semigroup of $R$, and it is easily seen that it is the supremum of $\mathcal{T}$ in $\mathcal{S}(R)$, with the order induced by the hull-kernel topology, that is the set theoretic inclusion.

For investigating the existence of the infimum of $\mathcal{T}$, we cannot argue in a dual way, since the natural candidate $\bigcap\left\{\mathscr{Q}_{\alpha} \mid \alpha \in A\right\}$ is not, in general, a prime semigroup (for example, if $P$ and $Q$ are incomparable prime ideals of $R$, they are both prime semigroups, but $P \cap Q$ is not). However, we can show that an infimum can be determined in many cases. More precisely, let $j: \mathcal{S}(R) \hookrightarrow \mathcal{X}(R)$ be the topological embedding defined in Proposition 2.7(1). Then, the set $j(\mathcal{T}):=\left\{j\left(\mathscr{Q}_{\alpha}\right) \mid\right.$ $\alpha \in A\}$ is a family of closed subsets in the inverse topology of $\operatorname{Spec}(R)$, and so if $C_{\mathcal{T}}:=\bigcap\left\{j\left(\mathscr{Q}_{\alpha}\right) \mid \alpha \in A\right\}$ is nonempty (for instance, since $X^{\text {inv }}$ is compact, for this assumption it suffices that the set of the $j\left(\mathscr{Q}_{\alpha}\right)$ satisfies the finite intersection property), then it still belongs to $\mathcal{X}(R)$, and it is the infimum of $j(\mathcal{T})$ in $\mathcal{X}(R)$. We claim that $C_{\mathcal{T}}=j\left(\mathscr{Q}_{0}\right)$ for some $\mathscr{Q}_{0} \in \mathcal{S}(R)$. More precisely, we claim that $\mathscr{Q}_{0}=\bigcup\left\{Q \mid Q \in C_{\mathcal{T}}\right\}$. 
Indeed, if $Q \in C_{\mathcal{T}}$ then $Q \in j\left(\mathscr{Q}_{0}\right)$ by Proposition 2.7(1). Conversely, if $P \in$ $j\left(\mathscr{Q}_{0}\right)$, then $P \subseteq \mathscr{Q}_{0} \subseteq \mathscr{Q}_{\alpha}$ for every $\alpha \in A$, and thus (again, by Proposition 2.7(1)) $P \in j\left(\mathscr{Q}_{\alpha}\right)$ for every $\alpha$, i.e., $P \in C_{\mathcal{T}}$. Therefore, $j\left(\mathscr{Q}_{0}\right)$ is the infimum of $j(\mathcal{T})$ in $j(\mathcal{S}(R))$, and since $j$ is a homeomorphism between $\mathcal{S}(R)$ and its image in $\mathcal{X}(R)$, it follows that $\mathscr{Q}_{0}$ is the infimum of $\mathcal{T}$ in $\mathcal{S}(R)$.

(b) From (a), it follow by construction that the topological embedding $j$ : $\mathcal{S}(R) \hookrightarrow \mathcal{X}(R)$ preserves the infimum, in the cases where it exists. However, the embedding $j$ in general does not preserve the supremum.

For example, let $D$ be a local unique factorization domain of dimension 2 , and let $Y_{1}, Y_{2}$ be two nonempty disjoint sets of prime ideals such that $Y_{1} \cup Y_{2}$ is the set $\operatorname{Spec}^{1}(D)$ of prime ideals of height 1 of $D$. If $\mathscr{Q}_{i}:=\bigcup\left\{P \mid P \in Y_{i}\right\}$, then $j\left(\mathscr{Q}_{i}\right)=$ $Y_{i} \cup\{(0)\}$, and thus $j\left(\mathscr{Q}_{1}\right) \cup j\left(\mathscr{Q}_{2}\right)=\operatorname{Spec}^{1}(D) \cup\{(0)\} \subsetneq \operatorname{Spec}(D)$. However, $\mathscr{Q}_{1} \cup \mathscr{Q}_{2}$ is equal to the set of non-units of $D$, so that $j\left(\mathscr{Q}_{1} \cup \mathscr{Q}_{2}\right)=\operatorname{Spec}(D)$.

On the other hand, if $\left\{P_{1}, P_{2}, \ldots, P_{n}\right\}$ is a finite set of prime ideals (and thus, in particular, of prime semigroups) of $R$, then $j\left(P_{1} \cup P_{2} \cup \cdots \cup P_{n}\right)=j\left(P_{1}\right) \cup j\left(P_{2}\right) \cup$ $\cdots \cup j\left(P_{n}\right)$. Indeed, by Proposition 2.7(1), $Q \in j\left(P_{1} \cup P_{2} \cup \cdots \cup P_{n}\right)$ if and only if $Q \subseteq P_{1} \cup P_{2} \cup \cdots \cup P_{n}$ and, by prime avoidance, this is equivalent to $Q \subseteq P_{i}$ for some $i$, and thus to $Q \in j\left(P_{i}\right)$ for some $i$.

\section{The integral DOMAIN CASE}

Let $D$ be an integral domain, and recall that the set $\operatorname{Dverr}(D)$ of the overrings of $R$ has a natural topological structure (see Section [1.6). Then, there is a natural map

$$
\begin{aligned}
\ell_{0}: \operatorname{Spec}(D) & \longrightarrow \operatorname{Overr}(D) \\
P & \longmapsto D_{P},
\end{aligned}
$$

which is a topological embedding [9, Lemma 2.4]. We show next that $\mathcal{S}(D)$ admits a similar interpretation with respect to $\operatorname{Dverr}(D)$.

Proposition 3.1. Let $D$ be an integral domain with quotient field $K$ and let $\operatorname{Derr}(D)$ be the set of the overrings of $D$, endowed with the Zariski topology.

(1) Let $\mathscr{Q} \in \mathcal{S}(D)$ and set as above $\Sigma_{\mathscr{Q}}:=D \backslash \mathscr{Q}$ and $D_{\mathscr{Q}}:=\Sigma_{\mathscr{Q}}^{-1} D$. The map

$$
\begin{aligned}
\ell: \mathcal{S}(D) & \longrightarrow \text { Overr }(D) \\
\mathscr{Q} & \longmapsto D_{\mathscr{Q}}
\end{aligned}
$$

is a topological embedding that extends the map $\ell_{0}$ defined above.

(2) The map

$$
\begin{aligned}
& \omega: \operatorname{Overr}(D) \longrightarrow \mathcal{X}(D) \\
& T \longmapsto \mathrm{QSpec}^{\widetilde{\wedge_{\{T\}}}}(D)
\end{aligned}
$$

is a continuous map of spectral spaces. Moreover, if $T \in \operatorname{Overr}(D)$ and the canonical embedding $\tau: D \longrightarrow T$ is flat, then $\omega(T)=\tau^{a}(\operatorname{Spec}(T))$.

(3) The composition $\omega \circ \ell: \mathcal{S}(D) \hookrightarrow \mathcal{X}(D)$ coincides with the topological embedding $j$ defined in Proposition 2.7(1).

Proof. (1) Since $\{\mathrm{B}(x) \mid x \in K\}$ is a subbasis of open sets for Overr $(D)$, to get continuity of $\ell$ it suffices to prove that, if $x \in K$, then $\ell^{-1}(\mathrm{~B}(x))$ is open in $\mathcal{S}(D)$. Take a semigroup prime $\mathscr{Q} \in \ell^{-1}(\mathrm{~B}(x))$, and let $d, s \in D$ with $s \notin \mathscr{Q}$ such that 
$x=\frac{d}{s} \in D_{\mathscr{Q}}$. Then, we have $\mathscr{Q} \in \boldsymbol{U}(s) \subseteq \ell^{-1}\left(\mathrm{~B}\left(s^{-1}\right)\right) \subseteq \ell^{-1}(\mathrm{~B}(x))$, that is, $\ell^{-1}(\mathrm{~B}(x))$ is open in $\mathcal{S}(D)$.

To prove that $\ell$ is a topological embedding it is now sufficient to note that, for any nonzero element $f \in D$, we have $\ell(\boldsymbol{U}(f))=\ell(\mathcal{S}(D)) \cap \mathrm{B}\left(f^{-1}\right)$. The inclusion $\subseteq$ is trivial. Conversely, if $T \in \ell(\mathcal{S}(D)) \cap \mathrm{B}\left(f^{-1}\right)$, then there are a semigroup prime $\mathscr{Q}$ and elements $d, s \in D$ such that $s \notin \mathscr{Q}$ and $\frac{1}{f}=\frac{d}{s} \in D_{\mathscr{Q}}=T$. It follows $s=d f \notin \mathscr{Q}$ and, a fortiori, by definition of semigroup prime, $f \notin \mathscr{Q}$. Then $T \in \ell(\boldsymbol{U}(f))$, and thus the proof is complete.

(2) It is sufficient to note that $\omega$ is the composition of three continuous maps, namely the topological embedding $\iota: \operatorname{Overr}(D) \hookrightarrow \operatorname{SStar}(D)$ (defined, for each overring $T$ of $D$, by $\iota(T):=\wedge_{\{T\}}$ [21, Proposition 2.5]), the continuous surjection $\widetilde{\Phi}: \operatorname{SStar}(D) \rightarrow \widetilde{\operatorname{SStar}}(D)$ (defined, for each $\star \in \operatorname{SStar}(D)$, by $\widetilde{\Phi}(\star):=\widetilde{\text { S }}[19$, Proposition 4.3(2)]), and the homeomorphism $\Delta: \widetilde{\operatorname{SStar}}(D) \stackrel{\sim}{\rightarrow} \mathcal{X}(D)$ (defined, for each $\star \in \widetilde{\operatorname{SStar}}(D)$, by $\Delta(\star):=\operatorname{QSppec}^{\star}(D)$ [20, Proposition 5.2(1)]).

Suppose $T$ is flat over $D$. In order to show that $\operatorname{QSpec}^{\wedge_{\{T\}}}(D)=\{Q \cap D \mid Q \in$ $\operatorname{Spec}(T)\}$ we observe that, even if $T$ is not $D$-flat, the equality QSpec $\widetilde{\wedge_{\{T\}}}(D)=$ $\mathrm{Cl}^{\text {inv }}\left(\mathrm{QSpec}^{\wedge}{ }_{\{T\}}(D)\right)$ holds, in view of Remark 1.1] since $\wedge_{\{T\}}$ is a semistar operation of finite type. Moreover, $P \in \mathrm{QSpec}^{\wedge}{ }_{\{T\}}(D)$ if and only if $P=P T \cap D$. Hence, $\tau^{a}(\operatorname{Spec}(T))=\{Q \cap D \mid Q \in \operatorname{Spec}(T)\} \subseteq \mathrm{QSpec}^{\wedge}{ }_{\{T\}}(D)$. Conversely, assuming that $T$ is $D$-flat, if $P \in \operatorname{QSpec}^{\wedge}\{T\}(D)$ and if $Q \in \operatorname{Spec}(T)$ and it is minimal over $P T$ then, by flatness, $Q \cap D=P$ [39, Section 1-6, Exercise 37] and so $P \in \tau^{a}(\operatorname{Spec}(T))$.

(3) is a straightforward consequence of the definitions.

When we specialize our investigation to the class of Prüfer domains, we obtain more precise statements.

Proposition 3.2. Let $D$ be a Prüfer domain. Then, the chain of canonical maps

$$
\text { Overr }(D) \stackrel{\iota}{\longrightarrow} \operatorname{SStar}_{f}(D) \stackrel{\widetilde{\Phi}}{\longrightarrow} \widetilde{\operatorname{SStar}}(D) \stackrel{\Delta}{\longrightarrow} \mathcal{X}(D)
$$

is a chain of homeomorphisms, and $\widetilde{\Phi}$ is the identity. Moreover, the composition $\Delta \circ \widetilde{\Phi} \circ \iota$ coincides with the map $\omega$ defined in Proposition [3.1(2), and $\omega(T):=$ $\operatorname{QSpec}^{\wedge}\{T\}(D)$ for all $T \in \operatorname{Overr}(D)$.

Proof. The map $\Delta: \widetilde{\operatorname{SStar}}(D) \rightarrow \mathcal{X}(D)$ (defined by $\Delta(\star):=\mathrm{QSpec}^{\star}(D)$ for each $\star$ spectral semistar operation of finite type on $D)$ is a homeomorphism by 20 , Proposition 5.2(1)].

Since $D$ is a Prüfer domain, each of its overrings is $D$-flat [23, Theorem 1.1.1]. Then, the canonical map $\widetilde{\Phi} \circ \iota: \operatorname{Dverr}(D) \longrightarrow \widetilde{\operatorname{SStar}}(D), T \mapsto \wedge_{\{T\}}=\widetilde{\Lambda_{\{T\}}}$, is a topological embedding (proof of Proposition 3.1(2) or [21, Proposition 2.5]).

We need to show that $\operatorname{SStar}_{f}(D)=\widetilde{\operatorname{SStar}}(D)$. Indeed, if $\star \in \operatorname{SStar}_{f}(D)$, then the domain $D^{\star}$, as an overring of a Prüfer domain, is still a Prüfer domain. Hence, $\wedge_{\left\{D^{\star}\right\}}=\widetilde{\Lambda_{\left\{D^{\star}\right\}}}$, since $D^{\star}$ is $D$-flat, and $D^{\star}$ admits a unique star operation of finite type. It follows that $\left.\star\right|_{\boldsymbol{F}\left(D^{\star}\right)}: \boldsymbol{F}\left(D^{\star}\right) \rightarrow \boldsymbol{F}\left(D^{\star}\right)$ is the identity star operation of $D^{\star}$. On the other hand note that, for each $F \in \boldsymbol{f}(D)$,

$$
F^{\star}=(F D)^{\star}=\left(F D^{\star}\right)^{\star}=F D^{\star} .
$$

Therefore, we have $\star=\wedge_{\left\{D^{\star}\right\}}$ and so $\iota$ is surjective. 
The equality $\omega=\Delta \circ \widetilde{\Phi} \circ \iota$ holds in general (see the proof of Proposition 3.1 (2)) and the last claim follows from the fact that $\wedge_{\{T\}}=\widetilde{\wedge_{\{T\}}}$, since every overring $T$ of the Prüfer domain $D$ is $D$-flat.

Recall that an integral domain $D$ is a $Q R$-domain if each overring of $D$ is a ring of fractions of $D$ (for more details see, for example, 29] and [35]). For example, a Bézout domain is a QR-domain [28, page 250 Exercise 10(b)].

Corollary 3.3. Let $D$ be a $Q R$-domain. Then, the chain of maps

$$
\mathcal{S}(D) \stackrel{\ell}{\longrightarrow} \operatorname{Overr}(D) \stackrel{\omega}{\longrightarrow} \mathcal{X}(D)
$$

is a chain of homeomorphisms.

Proof. By Proposition 2.7(3), $\ell$ is a topological embedding, and the hypothesis that $D$ is a QR-domain guarantees that $\ell$ is also surjective. Therefore, $\ell$ is a homeomorphism. Since a QR-domain is - in particular - a Prüfer domain [28, p. 334], then we know from Proposition 3.2 that $\omega$ is a homeomorphism. The claim follows.

Example 3.4. Consider a Dedekind domain $D$ such that the class group $\mathrm{Cl}(D)$ of $D$ is not a torsion group (an explicit example is given by $D:=K[X, Y] /\left(X^{2}-\right.$ $\left.Y^{3}+Y+1\right)$, where $K$ is an algebraically closed field; see [29, Sections 3 and 4$]$ and [52, page 146], and for a general result [27, Theorem 14.10]). Then, there is a maximal ideal $P$ of $D$ such that the class $[P]$ has infinite order in $\mathrm{Cl}(D)$, i.e., $P^{n}$ is never principal or, equivalently, no principal ideal is $P$-primary [27, Proposition 6.8]. Let $Y:=\operatorname{Spec}(D) \backslash\{P\}$ : then, $Y$ is closed in the inverse topology, since it is a quasi-compact open subspace of $\operatorname{Spec}(D)$, endowed with the Zariski topology. We claim that $Y \notin j(\mathcal{S}(D))$. If it was, say $Y=j(\mathscr{Q})$, then $\mathscr{Q} \in \mathcal{S}(D)$ must contain every element of $Y$, but there must be an $x \in P$ such that $x \notin \mathscr{Q}$. However, the ideal $x D$ is not $P$-primary, and so there also exists a prime ideal $Q$ of $D, Q \neq P$, such that $x \in Q$. This contradicts $Y=j(\mathscr{Q})$, and so $j$ is not surjective.

On the other hand, if $D^{\prime}$ is a principal ideal domain, then $j^{\prime}: \mathcal{S}\left(D^{\prime}\right) \rightarrow \mathcal{X}\left(D^{\prime}\right)$ is surjective (Corollary 3.3). Moreover, we can always find a principal ideal domain $D^{\prime}$ such that the cardinality of $\operatorname{Max}\left(D^{\prime}\right)$ is equal to the cardinality of $\operatorname{Max}(D)$ (it suffices to take $D^{\prime}:=F[T]$, where $F$ is a field with the same cardinality of $\operatorname{Max}(D)$ ) and $T$ is an indeterminate over $F$. Then, $\operatorname{Spec}\left(D^{\prime}\right)$ and $\operatorname{Spec}(D)$ are homeomorphic (it is enough to take any bijection between $\operatorname{Max}\left(D^{\prime}\right)$ and $\operatorname{Max}(D)$ then extend it to a bijection $\rho: \operatorname{Spec}\left(D^{\prime}\right) \rightarrow \operatorname{Spec}(D)$ such that $\left.\rho((0))=(0)\right)$, but $j^{\prime}$ is surjective while $j$ is not.

Remark 3.5. Note that, by [53, Theorem 2.2], when $R:=D$ is a Dedekind domain, the condition that the canonical map $\mathcal{S}(D) \rightarrow \mathcal{X}(D)$ is a homeomorphism and, hence, the equivalent conditions of Corollary 2.14 are equivalent to the following:

(iv) The ideal class group of $D$ is torsion.

(v) $D$ is a QR-domain.

\section{The Space of Semigroup Primes of the Nagata Ring}

Our next goal is to show that, for each ring $R$, the spectral space $\mathcal{X}(R)$ can be embedded in a space of prime semigroups of a different ring $A$ : more precisely, we will show that we can choose $A$ to be the Nagata ring of $R$. 
Recall that, given a ring $R$ and an indeterminate $T$ over $R$, the Nagata ring $R(T)$ of $R$ is the localization $S^{-1} R[T]$, where $S$ is the multiplicative set of all the primitive polynomials of $R[T]$. It is well known [28, Proposition 33.1(1)] that $S=R[T] \backslash \bigcup\{M[T] \mid M \in \operatorname{Max}(R)\}$. Let $g: R \hookrightarrow R(T)$ be the canonical embedding. For the sake of simplicity, we identify $R$ with $g(R)$ inside $R(X)$. It is clear that the spectral map $g^{a}: \operatorname{Spec}(R(T)) \rightarrow \operatorname{Spec}(R)$ is surjective. For uses of Nagata rings and related rings of rational functions in the context of star and semistar operations, see [28, [24], [25], [4], [5], 66, [7, [12], 334, [38], [37] and [45].

Now, we consider another map $\gamma: \operatorname{Spec}(R) \rightarrow \operatorname{Spec}(R(T))$ by setting $\gamma(P):=$ $P R(T)$ for each $P \in \operatorname{Spec}(R)$ : this map is well-defined and injective (since $I R(T) \cap$ $R=I$, for all ideals $I$ of $R$ [28, Proposition 33.1(4)]). Clearly, $\gamma \circ g^{a}$ is the identity map of $\operatorname{Spec}(R)$. Further properties are given next.

Lemma 4.1. Let $\gamma: \operatorname{Spec}(R) \rightarrow \operatorname{Spec}(R(T))$ and $g^{a}: \operatorname{Spec}(R(T)) \rightarrow \operatorname{Spec}(R)$ be as above.

(1) The map $\gamma$ is a spectral embedding and $g^{a}$ is a spectral retraction.

Let $Y$ and $Z$ two nonempty subsets of $\operatorname{Spec}(R)$, and, for any $X \subseteq \operatorname{Spec}(R)$, let $\mathscr{Q}(X):=\bigcup\{P R(T) \mid P \in X\} \subseteq R(T)$.

(2) If $\mathrm{Cl}^{\text {inv }}(Y)=\mathrm{Cl}^{\text {inv }}(Z)$, then also $\mathrm{Cl}^{\text {inv }}(\gamma(Y))=\mathrm{Cl}^{\text {inv }}(\gamma(Z))$.

(3) The equality $\mathscr{Q}(Y)=\mathscr{Q}(Z)$ holds if and only if $\mathrm{Cl}^{\mathrm{inv}}(Y)=\mathrm{Cl}^{\mathrm{inv}}(Z)$.

Proof. (1) Take a nonzero element $f / p \in R(T)$, where $f, p \in R[T]$ and $p$ is primitive, and write $f:=a_{0}+a_{1} T+\ldots+a_{n} T^{n}$. For any prime ideal $P$ of $R$, we have:

$$
\frac{f}{p} \notin P R(T) \Longleftrightarrow f \notin P R[T] \Longleftrightarrow P \nsupseteq\left(a_{0}, a_{1} \ldots, a_{n}\right) R,
$$

that is, $\gamma^{-1}\left(\mathrm{D}\left(\frac{f}{p} R(T)\right)\right)=\mathrm{D}\left(\left(a_{0}, a_{1}, \ldots, a_{n}\right) R\right)$. This proves that $\gamma$ is (continuous and) spectral. Moreover, for each $x \in R$ we have $\gamma(\mathrm{D}(x R))=\mathrm{D}(x R(T)) \cap \operatorname{Im}(\gamma)$, and thus $\gamma$ is a topological embedding. The conclusion follows from the fact that $g^{a} \circ \gamma$ is the identity of $\operatorname{Spec}(R)$.

(2) Assume that $\mathrm{Cl}^{\text {inv }}(Y)=\mathrm{Cl}^{\text {inv }}(Z)$. By definition, a basis for closed sets for the inverse topology of $\operatorname{Spec}(R(T))$ is given by the quasi-compact open subspaces of $\operatorname{Spec}(R(T)$ ) (when endowed with the Zariski topology). Thus, we have to prove that, for any nonzero finitely generated ideal $J$ of $R(T)$, we have $\gamma(Y) \subseteq \mathrm{D}(J)$ if and only if $\gamma(Z) \subseteq \mathrm{D}(J)$. Let $\frac{f_{1}}{p_{1}}, \frac{f_{2}}{p_{2}}, \ldots, \frac{f_{r}}{p_{r}} \in R(T)$ be generators of the ideal $J$, where $f_{i}, p_{i} \in R[T]$ and $p_{i}$ is primitive, for $i=1,2, \ldots, r$, and let $C_{i}$ be the content of $f_{i}$. Then $\gamma(Y) \subseteq \mathrm{D}(J)$ if and only if for any $P \in Y$ there is some index $i$ such that $\frac{f_{i}}{p_{i}} \notin P R(T)$, that is $f_{i} \notin P R[T]=P R(T) \cap R[T]$. In other words, $P \nsupseteq C_{i}$, i.e., $P \in \mathrm{D}\left(C_{i}\right)$. If we set $C:=C_{1}+C_{2}+\ldots+C_{r}$, the previous argument shows that $\gamma(Y) \subseteq \mathrm{D}(J)$ if and only if $Y \subseteq \mathrm{D}(C)$. Since $C$ is a finitely generated ideal of $R$, the set $D(C)$ is a quasi-compact open subspace of $\operatorname{Spec}(R)$, and thus also $Z \subseteq \mathrm{D}(C)$, because $Y$ and $Z$ have the same closure, with respect to the inverse topology. Thus, any prime ideal $Q$ of $Z$ does not contain some coefficient of some polynomial $f_{i}$, and then $\frac{f_{i}}{p_{i}} \notin Q R(T)$, that is $\gamma(Z) \subseteq \mathrm{D}(J)$.

(3) If $\mathrm{Cl}^{\text {inv }}(Y)=\mathrm{Cl}^{\text {inv }}(Z)$ then $\mathrm{Cl}^{\text {inv }}(\gamma(Y))=\mathrm{Cl}^{\text {inv }}(\gamma(Z))$, by part $(2)$. Thus, the equality $\mathscr{Q}(Y)=\mathscr{Q}(Z)$ holds by Remark 2.12, Conversely, assume that $\mathscr{Q}(Y)=$ $\mathscr{Q}(Z)$, and let $J:=\left(a_{0}, a_{1}, \ldots, a_{n}\right) R$ be a nonzero finitely generated ideal of $R$. We have to prove that $Y \subseteq \mathrm{D}(J)$ if and only if $Z \subseteq \mathrm{D}(J)$. Suppose that $Y \subseteq \mathrm{D}(J)$. 
Then, if $f:=a_{0}+a_{1} T+\ldots+a_{n} T^{n} \in R[T]$, we have $f \notin P R[T]=P R(T) \cap R[T]$, for each $P \in Y$, that is $\frac{f}{1} \notin \mathscr{Q}(Y)=\mathscr{Q}(Z)$. In other words, $f \notin Q R[T]$, for each $Q \in Z$, i.e., $Z \subseteq \mathrm{D}(J)$.

Now, we are in condition to prove that the spectral space $\mathcal{X}(R)$ can be embedded in the spectral space of prime semigroups of the Nagata ring $R(T)$.

Proposition 4.2. Let $R$ be a ring, $j: \mathcal{S}(R) \hookrightarrow \mathcal{X}(R)$ the spectral embedding defined in Proposition 2.7(1), $g: R \hookrightarrow R(T)$ the canonical ring embedding and let $\mathcal{S}(g): \mathcal{S}(R(T)) \rightarrow \mathcal{S}(R)$ be the spectral map associated to $g$ defined in (11). Define $\boldsymbol{\nu}$ as the map

$$
\begin{aligned}
\boldsymbol{\nu} \mathcal{X}(R) & \longrightarrow \mathcal{S}(R(T)) \\
Y & \longmapsto \bigcup\{P R(T) \mid P \in Y\} .
\end{aligned}
$$

The following properties hold.

(1) $\boldsymbol{\nu}$ is a spectral embedding.

(2) $\mathcal{S}(g) \circ \boldsymbol{\nu} \circ j$ is the identity of $\mathcal{S}(R)$. In particular, $\mathcal{S}(g): \mathcal{S}(R(T)) \rightarrow \mathcal{S}(R)$ is a topological retraction.

(3) If $\mathscr{P}: \mathcal{X}(R) \rightarrow \mathcal{S}(R)$ is the map defined in Proposition [2.11, then $\mathscr{P}=$ $\mathcal{S}(g) \circ \boldsymbol{\nu}$

Proof. (1). By Lemma 4.1(3), the map $\boldsymbol{\nu}$ is injective. Now, let $0 \neq \frac{f}{p} \in R(T)$, where $f, p \in R[T]$ and $p$ is primitive and let $C$ be the content of the polynomial $f$. Then, using the notation of Lemma 4.1(3),

$$
\begin{aligned}
\boldsymbol{\nu}^{-1}\left(\boldsymbol{U}\left(\frac{f}{p}\right)\right) & =\left\{Y \in \mathcal{X}(R) \mid \frac{f}{p} \notin \mathscr{Q}(Y)\right\} \\
& =\{Y \in \mathcal{X}(R) \mid f \notin P R[T] \text { for all } P \in Y\}=\mathcal{U}(\mathrm{D}(C)) .
\end{aligned}
$$

This proves that $\boldsymbol{\nu}$ is continuous and spectral. On the other hand, with similar arguments, it can be shown that, given $a_{0}, a_{1}, \ldots, a_{n} \in R$, if $f:=a_{0}+a_{1} T+\ldots+$ $a_{n} T^{n} \in R[T]$ we have

$$
\boldsymbol{\nu}\left(\mathrm{D}\left(a_{0}, a_{1}, \ldots a_{n}\right)\right)=\operatorname{Im}(\boldsymbol{\nu}) \cap \boldsymbol{U}\left(\frac{f}{1}\right),
$$

that is, $\boldsymbol{\nu}$ is a topological embedding.

(2) Let $\mathscr{P} \in \mathcal{S}(R)$. Let $Y$ be a nonempty set of prime ideals of $R$ such that $\mathscr{P}=\mathscr{P}(Y)=\bigcup\{P \in \operatorname{Spec}(R) \mid P \in Y\}$ (Lemma 2.2). Set $\mathscr{Q}(Y):=\bigcup\{P R(T) \mid$ $P \in Y\} \in \mathcal{S}(R(T))$. Recall that, for each prime ideal $P \in \operatorname{Spec}(R), P R(T) \cap R=$ $g^{-1}(P R(T))=P[28$, Proposition 33.1(4)]. Then,

$$
\begin{aligned}
\mathcal{S}(g) \circ \boldsymbol{\nu} \circ j(\mathscr{P}) & =\mathcal{S}(g)(\mathscr{Q}(Y))=g^{-1}(\mathscr{Q}(Y)) \\
& =(\bigcup\{P R(T) \mid P \in Y\}) \cap R \\
& =\bigcup(\{P R(T) \mid P \in Y\} \cap R) \\
& =\bigcup\{P \in \operatorname{Spec}(R) \mid P \in Y\}=\mathscr{P} .
\end{aligned}
$$

(3) Let $Y \in \mathcal{X}(R)$. Then, we have

$$
(\boldsymbol{S}(g) \circ \boldsymbol{\nu})(Y)=g^{-1}(\boldsymbol{\nu}(Y))=g^{-1}\left(\bigcup_{P \in Y} P R(T)\right)=\bigcup_{P \in Y} g^{-1}(P R(T)) .
$$

However, as noted above, $g^{-1}(P R(T))=P$ for every $P \in \operatorname{Spec}(R)$, and thus $(\mathcal{S}(g) \circ \boldsymbol{\nu})(Y)=\bigcup\{P \mid P \in Y\}$, which is exactly the definition of $\mathscr{P}(Y)$. 
We now introduce some notation that will be used in the following Remark 4.3 and Proposition 4.4, where we will show that, given a $\operatorname{ring} R, \mathcal{X}(R)$ is a topological retract of the spectral space $\mathcal{S}(R(T))$.

If $\mathscr{Q} \in \mathcal{S}(R(T))$, then we set $\Sigma_{\mathscr{Q}}:=R(T) \backslash \mathscr{Q}, R(T)_{\mathscr{Q}}:=\Sigma_{\mathscr{Q}}^{-1} R(T)$. We denote by $g: R \rightarrow R(T)$ and $\lambda_{1}: R(T) \rightarrow R(T)_{\mathscr{Q}}$ the canonical flat homomorphisms and we set $\lambda:=\lambda_{1} \circ g: R \rightarrow R(T)_{\mathscr{Q}}$.

Remark 4.3. In [1] the authors introduced and studied what they called the flat topology on $\operatorname{Spec}(R)$, where $R$ is any ring, by taking as closed subspaces the subset $\rho^{a}\left(\operatorname{Spec}\left(R^{\prime}\right)\right)$ for $\rho: R \rightarrow R^{\prime}$ varying among the flat ring homomorphisms. By [11, Theorem 2.2] the flat topology on $\operatorname{Spec}(R)$ coincides with the inverse topology.

We are in condition to give an explicit description of the inverse-closed subspaces of $\operatorname{Spec}(R)$. Let $Y \subseteq \operatorname{Spec}(R)$, set as above $\mathscr{Q}(Y):=\bigcup\{P R(T) \mid P \in Y\} \in$ $\mathcal{S}(R(T))$. Then, it is straightforward to see that $\mathscr{Q}(Y)=\mathscr{Q}\left(\lambda^{a}\left(\operatorname{Spec}\left(R(T)_{\mathscr{Q}(Y)}\right)\right)\right.$, where $\lambda: R \rightarrow R(T)_{\mathscr{Q}(Y)}$ is the canonical flat embedding. Thus, in view of Lemma 4.1 (3) and of the fact that the image of $\lambda^{a}$ is closed in the inverse topology, being $\lambda$ flat, we have $\mathrm{Cl}^{\text {inv }}(Y)=\lambda^{a}\left(\operatorname{Spec}\left(R(T)_{\mathscr{Q}(Y)}\right)\right)$. In particular, $Y=\mathrm{Cl}^{\text {inv }}(Y)$ if and only if $Y=\lambda^{a}\left(\operatorname{Spec}\left(R(T)_{\mathscr{Q}(Y)}\right)\right)$.

Proposition 4.4. Let $R$ be a ring. With the notation introduced above, the map

$$
\begin{aligned}
\chi: \mathcal{S}(R(T)) & \longrightarrow \mathcal{X}(R) \\
\mathscr{Q} & \longmapsto \lambda^{a}\left(\operatorname{Spec}\left(R(T)_{\mathscr{Q}}\right)\right)
\end{aligned}
$$

is continuous and surjective. Moreover, if $\boldsymbol{\nu}: \mathcal{X}(R) \hookrightarrow \mathcal{S}(R(T))$ is the spectral embedding defined in Proposition 4.2(1), then $\boldsymbol{\chi} \circ \boldsymbol{\nu}$ is the identity on $\mathcal{X}(R)$.

Proof. Note that $\chi$ is well-defined by Remark 4.3. since, for any $\mathscr{Q} \in \mathcal{S}(R(T))$, the canonical homomorphism $\lambda: R \rightarrow R(T)_{\mathscr{Q}}$ is flat. Let $\mathcal{X}\left(g^{a}\right): \mathcal{X}(R(T)) \rightarrow \mathcal{X}(R)$ be the spectral map associated to the canonical flat ring embedding $g: R \rightarrow R(T)$ and defined by $\mathcal{X}\left(g^{a}\right)(Y):=g^{a}(Y)^{\text {gen }}=g^{a}(Y)=\left\{g^{-1}(Q) \mid Q \in Y\right\}=\{Q \cap R \mid Q \in$ $Y$ \}, for each $Y \in \mathcal{X}(R(T)$ ) (see [20, Proposition 4.1] and [11, Proposition 2.7]). Then, the map $\chi$ coincides with the composition of the topological embedding $j: \mathcal{S}(R(T)) \hookrightarrow \mathcal{X}(R(T))$ (Proposition 2.7(1)) with $\mathcal{X}\left(g^{a}\right)$. In fact,

$$
\begin{aligned}
\left(\mathcal{X}\left(g^{a}\right) \circ j\right)(\mathscr{Q}) & =\mathcal{X}\left(g^{a}\right)(\{Q \in \operatorname{Spec}(R(T)) \mid Q \subseteq \mathscr{Q}\}) \\
& =\mathcal{X}\left(g^{a}\right)\left(\lambda^{a}\left(\operatorname{Spec}(R(T))_{\mathscr{Q}}\right)\right)=g^{a}\left(\lambda^{a}\left(\operatorname{Spec}\left(R(T)_{\mathscr{Q}}\right)\right)\right) \\
& =\mathcal{X}\left(\lambda^{a}\right)\left(\operatorname{Spec}\left(R(T)_{\mathscr{Q}}\right)\right)=\lambda^{a}\left(\operatorname{Spec}\left(R(T)_{\mathscr{Q}}\right)=\chi(\mathscr{Q})\right) .
\end{aligned}
$$

Hence $\chi$ is continuous as a composition of continuous maps (Proposition 2.7(1) and 20. Proposition 4.1]).

Let now $Y \in \mathcal{X}(R)$. Set, as usual, $\mathscr{Q}(Y):=\bigcup\{P R(T) \mid P \in Y\}$. Then, a direct calculation shows that $(\boldsymbol{\chi} \circ \boldsymbol{\nu})(Y)$ is the canonical image of $\operatorname{Spec}\left(R(T)_{\mathscr{Q}(Y)}\right)$ into $\operatorname{Spec}(R)$, which is is clearly equal to $Y(\operatorname{Remark} 4.3)$. Therefore $\boldsymbol{\chi} \circ \boldsymbol{\nu}$ is the identity. This implies that $\chi$ is surjective.

Remark 4.5. Given a ring $R$, there is another possible natural way to define a continuous map $\mathcal{S}(R(T)) \longrightarrow \mathcal{X}(R)$. Indeed, define $\chi^{\prime}$ as the map

$$
\begin{aligned}
\chi^{\prime}: \mathcal{S}(R(T)) & \longrightarrow \mathcal{X}(R) \\
\mathscr{Q} & \longmapsto\{P \in \operatorname{Spec}(R) \mid g(P) \subseteq \mathscr{Q}\} .
\end{aligned}
$$




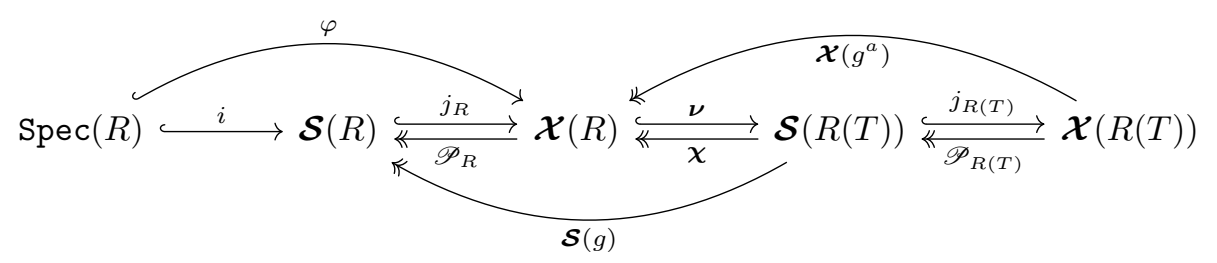

Figure 1. Maps between $\mathcal{S}$ - and $\mathcal{X}$-type spaces.

Clearly, $\chi(\mathscr{Q}) \subseteq \chi^{\prime}(\mathscr{Q})$, for each $\mathscr{Q} \in \mathcal{S}(R(T))$. Moreover, a direct calculation shows that $\chi^{\prime}=j \circ \mathcal{S}(g)$, so that $\chi^{\prime}$ is continuous. Furthermore, by Proposition $4.2(3)$, we have

$$
\chi^{\prime} \circ \boldsymbol{\nu}=j \circ \mathcal{S}(g) \circ \boldsymbol{\nu}=j \circ \mathscr{P} .
$$

Recall that $\boldsymbol{\chi} \circ \boldsymbol{\nu}$ is the identity on $\mathcal{X}(R)$ (Proposition 4.4) and, in general, $\boldsymbol{\chi}^{\prime} \circ \boldsymbol{\nu}(=$ $j \circ \mathscr{P}$ ) is not (Proposition 2.11(3)). We note that $\chi^{\prime}$, unlike $\chi$, is not surjective: for example, let $D$ be a 2 -dimensional Noetherian local ring and let $\operatorname{Spec}^{1}(D)$ be the set of the height-1 primes of $D$. Then, $Z:=\operatorname{Spec}^{1}(D) \cup\{(0)\}$ is inverse-closed in $\operatorname{Spec}(D)$, and the maximal ideal $M$ of $D$ is contained in the union of the elements of $Z$. Hence, $M D(T) \subseteq \mathscr{Q}(Z)$, and thus $M \in\{P \in \operatorname{Spec}(D) \mid g(P) \subseteq \mathscr{Q}(Z)\}=$ $\chi^{\prime}(\mathscr{Q}(Z))$. Therefore, $\chi^{\prime}(\mathscr{Q}(Z))=\operatorname{Spec}(D)$. On the other hand, $M \notin \chi(\mathscr{Q}(Z))$, since $Z=\chi(\mathscr{Q}(Z))$, because $Z$ is inverse-closed (Remark 4.3). We easily conclude that $Z$ is not in the range of $\chi^{\prime}$. As a matter of fact, suppose there exists a semigroup prime $\mathscr{Q}^{\star}$ of $D(T)$ such that $Z=\chi^{\prime}\left(\mathscr{Q}^{\star}\right)=\left\{P \in \operatorname{Spec}(D) \mid P \subseteq g^{-1}\left(\mathscr{Q}^{\star}\right)\right\}$. Thus, the union of all the prime ideals belonging to $Z$ is contained in $g^{-1}\left(\mathscr{Q}^{\star}\right)$ and, a fortiori, $M \subseteq g^{-1}\left(\mathscr{Q}^{\star}\right)$. It follows that $M \in \chi^{\prime}\left(\mathscr{Q}^{\star}\right)=Z$, a contradiction.

Acknowledgment. The authors thank the referee for his/her thorough report and highly appreciate the constructive comments and suggestions, which contributed to improving the quality of the paper.

\section{REFERENCES}

[1] D.D. Anderson, Star-operations induced by overrings, Comm. Algebra 16 (1988), 2535-2553.

[2] D.D. Anderson and D.F. Anderson, Examples of star operations on integral domains, Comm. Algebra 18 (1990), 1621-1643.

[3] D.D. Anderson and S.M. Clarke, Star-operations that distribute over finite intersections, Comm. Algebra 33 (2005), 2263-2274.

[4] G.W. Chang, *-Noetherian domains and the ring $D[X]_{N_{*}}$, J. Algebra 297 (2006), 216-233.

[5] G.W. Chang, Prüfer *-multiplication domains, Nagata rings, and Kronecker function rings, J. Algebra 319 (2008), 309-319.

[6] G.W. Chang, The Kronecker function ring of the ring $D[X]_{N_{*}}$, Bull. Korean Math. Soc. 47 (2010), 907-913.

[7] G.W. Chang and B.G. Kang, Prüfer-like domains and the Nagata ring of integral domains, Comm. Algebra 39 (2011), 4246-4258.

[8] C. Chevalley and H. Cartan, Schémas normaux; morphismes; ensembles constructibles, Séminaire Henri Cartan 8 (1955-1956), Exp. No. 7, 1-10.

[9] D.E. Dobbs, R. Fedder, and M. Fontana, Abstract Riemann surfaces of integral domains and spectral spaces. Ann. Mat. Pura Appl. (4), 148 (1987), 101-115.

[10] D.E. Dobbs and M. Fontana, Kronecker function rings and abstract Riemann surfaces, $J$. Algebra 226 (1986), 263-284.

[11] D.E. Dobbs, M. Fontana, and I.J. Papick, On the flat spectral topology. Rend. Mat. (7) 1 (1981), 559-578. 
[12] D. Dobbs and P. Sahandi, On semistar Nagata rings, Prüfer-like domains and semistar goingdown domains, Houston J. Math. 37 (2011), 715-731.

[13] J. Elliott, Functorial properties of star operations, Comm. Algebra 38 (2010), 1466-1490.

[14] N. Epstein, A guide to closure operations in commutative algebra, in "Progress in commutative algebra, Closures, Finiteness and Factorization", 2, (C. Francisco, L.C. Klingler, S.M. Sather-Wagstaff, and J. Vassilev, Editors), 1-37, Walter de Gruyter, Berlin, 2012.

[15] N. Epstein, Semistar operations and standard closure operations, Comm. Algebra 43 (2015), $325-336$.

[16] C.A. Finocchiaro, Spectral spaces and ultrafilters, Comm. Algebra 42 (2014), 1496-1508.

[17] C.A. Finocchiaro, M. Fontana, and K. A. Loper, The constructible topology on spaces of valuation domains, Trans. Am. Math. Soc. 365 (2013), 6199-6216.

[18] C.A. Finocchiaro, M. Fontana, D. Spirito, New distinguished classes of spectral spaces: a survey, in "Multiplicative Ideal Theory and Factorization Theory - Commutative and NonCommutative Perspectives" (S. Chapman, M. Fontana, A. Geroldinger, and B. Olberding, Editors), Springer Verlag Publisher 2016 (to appear).

[19] C.A. Finocchiaro, M. Fontana and D. Spirito, Spectral spaces of semistar operations, J. Pure. Appl. Algebra (2016, to appear).

[20] C.A. Finocchiaro, M. Fontana and D. Spirito, The space of inverse-closed subsets of a spectral space is spectral, submitted.

[21] C.A. Finocchiaro and D. Spirito, Some topological considerations on semistar operations, $J$. Algebra 409 (2014), 199-218.

[22] M. Fontana and J. Huckaba, Localizing systems and semistar operations, in "Non-Noetherian Commutative Ring Theory" (Scott T. Chapman and Sarah Glaz, Editors), Kluwer Academic Publishers, 2000, pp. 169-198.

[23] M. Fontana, J.A. Huckaba and I.J. Papick, Prüfer domains, M. Dekker, New York, 1997.

[24] M. Fontana and K.A. Loper, Nagata rings, Kronecker function rings and related semistar operations, Comm. Algebra 31 (2003), 4775-4805

[25] M. Fontana and K.A. Loper, An historical overview of Kronecker function rings, Nagata rings, and related star and semistar operations, in "Multiplicative Ideal Theory in Commutative Algebra: A Tribute to the Work of Robert Gilmer" (J.W. Brewer, S. Glaz, W. Heinzer, and B. Olberding, Editors), 169-187, Springer, New York 2006.

[26] M. Fontana and K. A. Loper, The patch topology and the ultrafilter topology on the prime spectrum of a commutative ring, Comm. Algebra 36 (2008), 2917-2922.

[27] R.M. Fossum, The divisor class group of a Krull domain, Springer, New York, 1973.

[28] R. Gilmer, Multiplicative Ideal Theory, M. Dekker, New York, 1972.

[29] R. Gilmer and J. Ohm, Integral domains with quotient overrings, Math. Ann. 153 (1964), $97-103$.

[30] H. B. Grimble, Prime Ideals in Semigroups, University of Tennessee, Knoxville, 1950.

[31] A. Grothendieck and J. Dieudonné, Éléments de Géométrie Algébrique I, IHES 1960; Springer, Berlin, 1970.

[32] F. Halter-Koch, Localizing systems, module systems, and semistar operations, J. Algebra 238 (2001), 723-761.

[33] F. Halter-Koch, Multiplicative ideal theory in the context of commutative monoids, in "Commutative algebra, Noetherian and non-Noetherian perspectives", 203-231, Springer, New York, 2011.

[34] F. Halter-Koch, Kronecker function rings and generalized integral closures, Comm. Algebra 31 (2003), 45-59.

[35] W. Heinzer, Quotient overrings of integral domains, Mathematika 17 (1970), 139-148.

[36] M. Hochster, Prime ideal structure in commutative rings, Trans. Amer. Math. Soc. 142 (1969), 43-60.

[37] P. Jara, Nagata rings, Front. Math. China 10 (2015), 91-110.

[38] B.G. Kang, Prüfer $v$-multiplication domains and the ring $R[X]_{N_{v}}$, J. Algebra 123 (1989), $151-170$.

[39] I. Kaplansky, Commutative rings, Allyn and Bacon, Boston, 1970.

[40] J. Kist, Minimal prime ideals in commutative semigroups, Proc. London Math. Soc. 13 (1963), 31-50. 
[41] K. A. Loper, The use of ultrafilters to study the structure of Prüfer and Prüfer-like rings, in "Commutative algebra, Noetherian and non-Noetherian perspectives" (M. Fontana, S.-E. Kabbaj, B. Olberding, I. Swanson, Editors), 319-333, Springer, New York, 2011.

[42] R. Matsuda, Note on spectral semistar operations of finite type, JP J. Algebra Number Theory Appl. 22 (2011), 225-231.

[43] J. Ohm and R.L. Pendleton, Rings with Noetherian spectrum, Duke Math. J. 35 (1968), 631-639.

[44] A. Okabe, A note on semistar operations and localizing systems, JP J. Algebra Number Theory Appl. 6 (2006), 149-174.

[45] A. Okabe and R. Matsuda, Kronecker function rings of semistar-operations, Tsukuba J. Math. 21 (1997), 529-540.

[46] A. Okabe and R. Matsuda, Semistar-operations on integral domains, Math. J. Toyama Univ. 17 (1994), 1-21.

[47] B. Olberding, Noetherian spaces of integrally closed rings with an application to intersections of valuation rings, Comm. Algebra 38 (2010), no. 9, 3318-3332.

[48] B. Olberding, Intersections of valuation overrings of two-dimensional Noetherian domains. in "Commutative algebra, Noetherian and non-Noetherian perspectives" (M. Fontana, S.-E. Kabbaj, B. Olberding, I. Swanson, Editors), 335-361, Springer, New York, 2011.

[49] B. Olberding, On the geometry of Prüfer intersections of valuation rings, Pacific J. Math. 273 (2015), 353-368.

[50] B. Olberding, Affine schemes and topological closures in the Zariski-Riemann space of valuation rings, J. Pure Appl. Algebra 219 (2015), 1720-1741.

[51] D. Rees, On semi-groups, Proc. Cambridge Phil. Society 36 (1940), 387-400.

[52] D. Rees, On a problem of Zariski, Illinois J. Math. 2 (1958), 145-149.

[53] C.M. Reis and T.M. Viswanathan, A compactness property for prime ideals in Noetherian rings, Proc. Amer. Math. Soc. 25 (1970), 353-356.

[54] R. Y. Sharp and P. Vámos, Baire's category theorem and prime avoidance in complete local rings, Archiv der Mathematik 44(3) (1985), 243-248.

[55] N. Schwartz and M. Tressl, Elementary properties of minimal and maximal points in Zariski spectra, J. Algebra 323 (2010), 698-728.

[56] W.W. Smith, A covering condition for prime ideals, Proc. Am. Math. Soc. 30 (1971), 451-452.

[57] M. H. Stone, Applications of the theory of Boolean rings to general topology, Trans. Amer. Math. Soc. 41 (1937), no. 3, 375-481.

[58] O. Zariski and P. Samuel, Commutative Algebra, Volume II, Van Nostrand, Princeton, 1960.

E-mail address:

finocchiaro@math.tugraz.at; fontana@mat.uniroma3.it; spirito@mat.uniroma3.it

C.A.F.: Institute of Analysis and Number Theory, University of Technology, Steyrergasse 30/II, 8010 Graz, Austria

M.F.\&D.S.: Dipartimento di Matematica e Fisica, Università degli Studi "Roma Tre", Largo San Leonardo Murialdo, 1, 00146 Roma, Italy 